\title{
AM-FM Energy Detection and Separation in Noise Using Multiband Energy Operators
}

\author{
Alan C. Bovik, Senior Member, IEEE, Petros Maragos, Senior Member, IEEE, \\ and Thomas F. Quatieri, Senior Member, IEEE
}

\begin{abstract}
This paper develops a multiband or wavelet approach for capturing the AM-FM components of modulated signals immersed in noise. The technique utilizes the recentlypopularized nonlinear energy operator $\Psi(s)=(s)^{2}-s \ddot{s}$ to isolate the AM-FM energy, and an energy separation algorithm (ESA) to extract the instantaneous amplitudes and frequencies. It is demonstrated that the performance of the energy operator/ ESA approach is vastly improved if the signal is first filtered through a bank of bandpass filters, and at each instant analyzed (via $\Psi$ and the ESA) using the dominant local channel response. Moreover, it is found that uniform (worst-case) performance across the frequency spectrum is attained by using a constant- $Q$, or multiscale wavelet-like filter bank.

The elementary stochastic properties of $\Psi$ and of the ESA are developed first. The performance of $\Psi$ and the ESA when applied to bandpass filtered versions of an AM-FM signal-plusnoise combination is then analyzed. The predicted performance Is greatly improved by filtering, if the local signal frequencies occur in-band. These observations motlvate the multiband energy operator and ESA approach, ensuring the in-band analysis of local AM-FM energy. In particular, the multi-bands must have the constant- $Q$ or wavelet scaling property to ensure uniform performance across bands. The theoretical predictions and the simulation results indicate that improved practical strategies are feasible for tracking and identifying AM-FM components in signals possessing pattern coherencies manifested as local concentrations of frequencies.
\end{abstract}

\section{INTRODUCTION}

$\mathrm{M}$ ETHODS for the accurate and efficient extraction of amplitude modulation (AM) and frequency modulation (FM) information in signals of the form

$$
s(t)=a(t) \cos [\phi(t)]
$$

Manuscript received September 1, 1992; revised June 10, 1993. The Guest Editor coordinating the review of this paper and approving it for publication was Dr. Ahmed Tewfik. This work was suppored in part by a University of Texas Faculty Research Assignment, in part by Texas Instruments under a grant, in part by the National Science Foundation under Grant MIP-91-20624, in part by the National Science Foundation Presidential Young Investigator Award under Grant MIP-86-58150 with matching funds from Xerox, and in part by the Naval Submarine Medical Research Laboratory.

A. C. Bovik is with the Department of Electrical and Computer Engineering, University of Texas at Austin, Austin. TX 78712-1084. Part of the research was conducted while he was on sabbatical at the Division of Applied Sciences, Harvard University, Cambridge, MA 02139.

P. Maragos is with the School of Electrical Engineering, Georgia Institute of Technology, Atlanta, GA 30332.

T. F. Quatieri is with the Lincoln Laboratory, Massachusetts Institute of Technology, Lexington, MA 02173

IEEE Log Number 9212179 are a topic of increased recent attention, owing to heightened interest in modulation models for e.g., speech signal production [1]- [3] and certain structures in optical images [4]. In (1), $s(t)$ has both time-varying amplitude $a(t)$ and time-varying instantaneous frequency

$$
\omega_{i}(t)=\dot{\phi}(t)
$$

where $\dot{\phi}=d \phi / d t$. Generally, the model (1) is most useful if $a(t)$ and $\omega_{i}(t)$ do not vary too rapidly, e.g., in the bandlimited sense [8].

The simple and elegant nonlinear signal operator

$$
\Psi(s)=(\dot{s})^{2}-s \ddot{s}
$$

developed by Teager [1], [2] and systematically introduced by Kaiser [5], [6], has been shown to be highly effective for detecting AM and FM modulation information in arbitrary AM-FM signals [8], in speech signals [7]-[9] and in its two-dimensional form, in image signals [10]. Indeed, for AM-FM signals of the form (1),

$$
\begin{aligned}
& \Psi(s) \approx a^{2}(t) \omega_{i}^{2}(t) \\
& \Psi(s) \approx a^{2}(t) \omega_{i}^{4}(t)
\end{aligned}
$$

with negligible approximation error under general realistic conditions [7]-[9]. This motivated the energy separation algorithm (ESA):

$$
\begin{aligned}
\hat{a}^{2}(t) & =\Psi^{2}(s) / \Psi(\dot{s}) \\
\hat{\omega}_{i}^{2}(t) & =\Psi(\dot{s}) / \Psi(s)
\end{aligned}
$$

as estimates of the squared amplitude envelope $a^{2}(t)$ and squared instantaneous frequency $\omega_{i}^{2}(t)$, respectively. Maragos, Kaiser, and Quatieri [7]-[9] have analyzed the efficacy of (4) and (5) in detail and have developed bounds on the absolute errors $|\hat{a}-a|$ and $\left|\hat{\omega}_{i}-\omega_{i}\right|$, which under general conditions are quite small [8], [9]. Note that in the case of a monochromatic signal ( $a=$ constant, $\omega_{i}=$ constant), (4) and (5) are exact.

In the current paper, the deterministic approximation errors in (4) and (5) are assumed small. Instead, the effects of noise and multiscale filtering on the behavior of the operator $\Psi$ and on the effectiveness of the ESA are considered. The effects of significant noise are very considerable-rendering $\Psi$ unpredictable and the ESA highly unreliable. However, the performance of the energy operator/ESA approach is vastly improved if the signal is 
first filtered through a bank of bandpass filters, and at each instant analyzed (via $\Psi$ and the ESA) using the dominant local channel response. Optimal performance is obtained when the filters are sufficiently narrowband (thus increasing the signal-to-noise ratio), the signal spectrum is sampled densely by the filter set (ensuring a high signal response in the analyzing channel), and importantly, by using a multiscale wavelet-like filter having the constant-Q property. Satisfying all of the prescriptions produces a multiband ESA having a great detal of noise resistance.

The remainder of the paper is organized as follows: In Section II, the basic statistical properties of the energy operator (3) are developed under the assumption that it is applied to a signal that is a zero-mean, wide-sense stationary (WSS) Gaussian random process. These results are then used in Section III, where approximate expressions are developed for the statistics of the output of the TeagerKaiser energy operator $\Psi$ when applied to an AM-FM signal of the form (1) immersed in noise. Section IV develops the statistical analysis of the ESA (4) and (5) using the signal-plus-noise approximation of Section III. A key element of these approximations is that there be an available narrowband channel filter that can effectively capture the local frequency structure of the noisy signal. In particular, as the analysis window is shifted over the signal, the local frequencies may sweep across the spectrum. This implies the necessity of a multiband filter implementation, where multiple bandpass filters densely sample the signal frequencies. It is also shown that consistent performance is achieved across low, high, and intermediate instantaneous frequencies, if the filter bank has the constant $Q$ property. These themes are developed in Section $\mathrm{V}$, where design criteria for the individual bandpass filters, and also for sampling the signal spectrum with multiple bandpass filters, are explored. Section VI develops some important examples, including analysis of the operation of the multiband ESA applied to the chirp signal in white noise. Extensive simulation results are also given in Section VI, which demonstrate the dramatic performance gains obtained using the multiband approach. The paper concludes in Section VII.

\section{Statistics of $\Psi$}

In this section the basic statistical (low-order moment) properties of the Teager-Kaiser energy operator (3) applied to a random signal $n(t)$ are developed. These properties prove to be fundamental in the analysis of systems that employ the Teager-Kaiser operator in the presence of noise.

Assume that $n(t)$ is a zero-mean, wide-sense stationary (WSS) Gaussian random process, with autocorrelation function $R(\tau)$ and power spectral density

$$
\Phi(\omega)=\int_{R} R(\tau) e^{-j \omega \tau} d \tau
$$

The assumption of (at least) wide-sense stationarity is necessary to develop nearly all of the properties in a useful form; without the assumption of Gaussianity, the analysis likewise becomes rapidly intractable. The assumption of a zero-mean is not critical, since the analysis is only slightly more complicated. In any case, nonzeromean (and nonstationary) signals expressed as the sum of a deterministic signal and a zero-mean WSS Gaussian process are considered later.

Since $n(t)$ is WSS Gaussian, the processes $\dot{n}(t)$ and $\ddot{n}(t)$ are also WSS Gaussian. Moreover, $\dot{n}(t)$ is statistically independent of both $n(t)$ and $\ddot{n}(t)$ [11]. Therefore, the energy operator output

$$
\Psi(n)=(\dot{n})^{2}-n \ddot{n}
$$

is the sum of two independent processes. Nevertheless, determining the probability density function of the process (6) is difficult, as it is the convolution of two rather complicated functions. Letting

$$
\begin{aligned}
& \operatorname{Var}[n]=R(0)=\gamma_{0}^{2} \\
& \operatorname{Var}[\dot{n}]=-R^{(2)}(0)=\gamma_{1}^{2} \\
& \operatorname{Var}[\ddot{n}]=R^{(4)}(0)=\gamma_{2}^{2} \\
& \mathrm{E}[n \ddot{n}]=R^{(2)}(0)=-\gamma_{1}^{2}
\end{aligned}
$$

where $E[\cdot]$ is the statistical expectation, $\operatorname{Var}[\cdot]$ is the variance, and

$$
R^{(k)}(\tau)=\frac{d^{k}}{d \tau^{k}} R(\tau)
$$

the probability density function of $(\dot{n})^{2}$ is then given by [11]

$w_{11}(b)= \begin{cases}\left(2 \pi b \gamma_{1}^{2}\right)^{-1 / 2} \exp \left[-b /\left(2 \gamma_{1}^{2}\right)\right], & b>0 \\ 0, & \text { else }\end{cases}$

while the probability density function of the product $n n$ is [12]

$w_{02}(b)=\pi^{-1} \Sigma_{02}^{-1 / 2} \exp \left(-b \gamma_{1}^{2} / \Sigma_{02}\right) K_{0}\left(|b| \gamma_{0} \gamma_{2} / \Sigma_{02}\right)$

for every $b$, where $\Sigma_{02}=\gamma_{0}^{2} \gamma_{0}^{2}-\gamma_{1}^{4}$ and $K_{0}(\cdot)$ is the modified Bessel function of the second kind and of order zero. The density function of $\Psi(n)$ is then

$$
w(b)=w_{11}(b) * w_{02}(b)
$$

where ' $*$ ' denotes linear convolution. In general, (9) cannot be expressed in a closed form, although the numerical evaluation of probabilities involving $\Psi(n)$ using (7)-(9) is straightforward.

Determining the moments of $\Psi(n)$ is much simpler. Indeed, we immediately have

$$
\begin{aligned}
\mathrm{E}[\Psi(n)] & =-2 R^{(2)}(0)=\frac{1}{\pi} \int_{R} \omega^{2} \Phi(\omega) d \omega \\
& =2 \gamma_{1}^{2} .
\end{aligned}
$$


Thus the expectation of the process $\Psi(n)$ is twice the variance of the process $\dot{n}(t)$-which is decidedly positive. Not so agreeable (although not surprising) is the fact that the mean of $\Psi(n)$ increases linearly with the spectral energy variance of $n(t)$. For a signal immersed in a significant noise element, this may pose severe problems unless steps are taken to ameliorate the noise.

The autocorrelation function $R_{\Psi(n)}(\tau)$ of $\Psi(n)$ can be easily found for the case of Gaussian $n(t)$ by using Isserlis's formula [13] to reduce all multiple-component moments:

$$
\begin{aligned}
R_{\Psi(n)}(\tau)= & 4\left[R^{(2)}(0)\right]^{2}+3\left[R^{(2)}(\tau)\right]^{2}+4 R^{(1)}(\tau) R^{(3)}(\tau) \\
& +R(\tau) R^{(4)}(\tau) .
\end{aligned}
$$

Hence from (10) and (11)

$$
\begin{aligned}
\operatorname{Var}[\Psi(n)]= & 3\left[R^{(2)}(0)\right]^{2}+R(0) R^{(4)}(0) \\
= & \left(\frac{1}{2 \pi}\right)^{2}\left\{3\left[\int_{R} \omega^{2} \Phi(\omega) d \omega\right]^{2}\right. \\
& \left.+\int_{R} \Phi(\omega) d \omega \int_{R} \omega^{4} \Phi(\omega) d \omega\right\} \\
= & 3 \gamma_{1}^{4}+\gamma_{0}^{2} \gamma_{2}^{2} .
\end{aligned}
$$

Thus, the variance of $\Psi(n)$ also increases dramatically with the presence of higher frequencies in the process $n(t)$. Furthermore, the inequality [11]

$$
\left[R^{(2)}(0)\right]^{2} \leq R(0) R^{(4)}(0)
$$

gives interesting bounds on the variance

$$
4\left[R^{(2)}(0)\right]^{2} \leq \operatorname{Var}[\Psi(n)] \leq 4 R(0) R^{(4)}(0)
$$

or equivalently

$$
\mathrm{E}^{2}[\Psi(n)] \leq \operatorname{Var}[\Psi(n)] \leq 4 \operatorname{Var}[n] \operatorname{Var}[\ddot{n}] .
$$

Since the lower bound in (13) is just $\mathrm{E}^{2}[\Psi(n)]$, then, although the expectation of $\Psi(n)$ may be positive, it is possible that $\Psi(n)$ may take negative values, which is highly undesirable. Not only does this suggest difficulty in evaluating the ESA of a signal immersed in noise, it also complicates the interpretation of $\Psi(n)$ as energy. For these reasons, positivity of the output of $\Psi$ has been explored in detail in [8], where sufficient conditions for positivity are given for narrowband AM-FM signals having limited amounts of amplitude/frequency modulation, and in [14], where necessary and sufficient conditions are given in terms of local geometric (convexity) properties of the signal.

Note that for an ideal bandpass process (i.e., $\Phi(\omega)=$ 0 whenever $\left.|\omega| \notin\left[\omega_{1}, \omega_{2}\right]\right)$, the following bounds [from (13), (14)] hold

$$
\begin{aligned}
& \omega_{1}^{2} \leq \frac{\mathrm{E}[\Psi(n)]}{2 R(0)} \leq \omega_{2}^{2} \\
& \omega_{1}^{4} \leq \frac{\operatorname{Var}[\Psi(n)]}{4 R^{2}(0)} \leq \omega_{2}^{4} .
\end{aligned}
$$

In the extreme case of a monochromatic process

$$
R(\tau)=A^{2} \cos \left(\omega_{0} \tau\right),
$$

it easily follows that

$$
\begin{aligned}
\mathrm{E}[\Psi(n)] & =2 A^{2} \omega_{0}^{2} \\
\operatorname{Var}[\Psi(n)] & =\mathrm{E}^{2}[\Psi(n)]=4 A^{4} \omega_{0}^{4} .
\end{aligned}
$$

Lastly, in evaluating the ESA (4) and (5) the energies of signal derivatives will also be required. Thus, the basic statistics of $\Psi(\dot{n})$ are of use. From the preceding discussion, it is easily established that

$$
\mathrm{E}[\Psi(\dot{n})]=2 R^{(4)}(0)
$$

and

$$
\begin{aligned}
R_{\Psi(n)}(\tau)= & 4\left[R^{(4)}(0)\right]^{2}+3\left[R^{(4)}(\tau)\right]^{2}+4 R^{(3)}(\tau) R^{(5)}(\tau) \\
& +R^{(2)}(\tau) R^{(6)}(\tau)
\end{aligned}
$$

hence,

$$
\operatorname{Var}[\Psi(\dot{n})]=3\left[R^{(4)}(0)\right]^{2}+R^{(2)}(0) R^{(6)}(0) .
$$

The cross-correlation properties of $\Psi(n)$ and $\Psi(\dot{n})$ can also be developed with a little effort. The cross-correlation is

$$
\mathrm{E}[\Psi(n) \Psi(\dot{n})]=-8 R^{(2)}(0) R^{(4)}(0),
$$

the cross-covariance is

$$
\operatorname{Cov}[\Psi(n), \Psi(\dot{n})]=-8 R^{(2)}(0) R^{(4)}(0),
$$

and the correlation coefficient between $\Psi(n)$ and $\Psi(\dot{n})$ is

$$
\begin{aligned}
\rho[\Psi(n), \Psi(i)]= & 4 \cdot\left[9+3 \frac{R(0) R^{(4)}(0)}{\left[R^{(2)}(0)\right]^{2}}+3\right. \\
& \left.\cdot \frac{R^{(2)}(0) R^{(6)}(0)}{\left[R^{(4)}(0)\right]^{2}}+\frac{R(0) R^{(6)}(0)}{R^{(2)}(0) R^{(4)}(0)}\right]^{-1 / 2}
\end{aligned}
$$

Thus, for the case of monochromatic process $(15), \Psi(n)$ and $\Psi(\dot{n})$ become linearly related

$$
\rho[\Psi(n), \Psi(\dot{n})]=1 .
$$

\section{ENergy of Bandpass-Filtered AM-FM Signals IN NOISE}

In this section the effect of additive noise on the response of the Teager-Kaiser energy operator applied to an AM-FM signal of the form (1) is analyzed. In particular, the degree to which noise effects can be ameliorated by bandpass filtering of the signal-plus-noise process is studied. Thus, consider the noise-corrupted AM-FM signal

$$
f(t)=s(t)+n(t)
$$

where the deterministic signal $s(t)$ is given by (1) and $n(t)$ is a zero-mean, WSS Gaussian random process with autocorrelation function $R(\tau)$ and power spectral density $\Phi(\omega)$ as in the preceding section.

Rather than studying the behavior of the energy $\Psi(f)$ 
of the combined process (21), instead consider a more general bandpass-filtered version of $(21) ; \Psi(f)$ may be analyzed as a special case using limiting arguments. Thus, define the linear bandpass filter with impulse response

$$
g_{\sigma}(t)=2 h_{\sigma}(t) \sin \left(\omega_{c} t\right)
$$

center freqency $\omega_{c}$, and frequency response

$$
G_{\sigma}(\omega)=\frac{1}{j}\left[H_{\sigma}\left(\omega-\omega_{c}\right)-H_{\sigma}\left(\omega+\omega_{c}\right)\right]
$$

where

$$
H_{\sigma}(\omega)=\frac{1}{\sqrt{\sigma}} H(\omega / \sigma),
$$

$H(\omega) \in L^{2}(R)$ is the frequency response of a low-pass filter with impulse response $h: \boldsymbol{R} \rightarrow \boldsymbol{R}$, and $\sigma>0$ is a parameter that scales bandwidth and center frequency. It is also assumed that $h(t)$ is even-symmetric, so that

$$
G_{o}(0)=0 \text {. }
$$

Define the kth-order spread of $H(\omega)$ about the value $\omega=$ $\pm \alpha$ to be

$$
V_{H}^{(k)}(\alpha)=\frac{1}{2 \pi}\left[\int_{R}(\omega \pm \alpha)^{2 k}|H(\omega)|^{2} d \omega\right]^{1 / 2 k}
$$

which is minimized at $\alpha=0$

$$
\begin{aligned}
V_{H}^{(1)}(0) & =\inf _{\alpha}\left\{V_{H}^{(k)}(\alpha)\right\} \\
& =\text { spectral energy variance of } H(\omega) .
\end{aligned}
$$

Note also that

$$
V_{H_{\sigma}}^{(1)}(\alpha)=\sigma V_{H}^{(1)}(\alpha / \sigma)
$$

so the bandwidth of $H_{\sigma}(\omega)$ is $\sigma$ times the bandwidth of $H(\omega)$. The filter energy, however, is constant across scales and is assumed to be unity

$$
\frac{1}{2 \pi} \int_{R}\left|H_{\sigma}(\omega)\right|^{2} d \omega=\frac{1}{2 \pi} \int_{R}|H(\omega)|^{2} d \omega=1
$$

For simplicity, it is assumed that for each combination of center frequency $\omega_{c}$ and parameter $\sigma$, the positive and negative frequency components of $G_{\sigma}(\omega)$ do not overlap

$$
\left|G_{\sigma}(\omega)\right|^{2}=\left|H_{\sigma}\left(\omega-\omega_{c}\right)\right|^{2}+\left|H_{\sigma}\left(\omega+\omega_{c}\right)\right|^{2} .
$$

Now denote the filtered signal-plus-noise combination

$$
f_{\sigma}(t)=s_{\sigma}(t)+n_{\sigma}(t)
$$

where $s_{\sigma}(t)=s(t) * g_{\sigma}(t)$ is the filtered signal and $n_{\sigma}(t)$ $=n(t) * g_{\mathfrak{o}}(t)$ the filtered noise process. The remainder of this section is devoted to analyzing the system depicted in Fig. 1. In Section III-A, approximate expressions are derived for the energy $\Psi\left(s_{\sigma}\right)$ of the filtered signal; and in Section III-B, for the moments of the energy $\Psi\left(n_{\sigma}\right)$ of the filtered noise process. In Section III-C, the statistics of the energy $\Psi\left(f_{\sigma}\right)$ of the filtered combination are analyzed utilizing the results of Sections III-A and III-B.

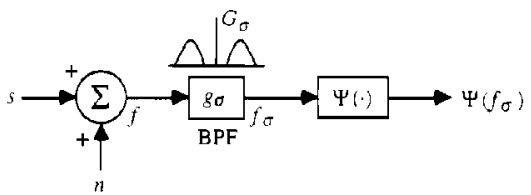

Fig. 1. Diagram of basic single-band energy operator.

\section{A. Filtered Signal Approximations}

An important approximation is made throughout this paper: if $s(t)=a(t) \cos [\phi(t)]$ is input to a linear system with frequency response $G_{\sigma}(\omega)$, then the response $s_{\sigma}(t)$ can be approximated by

$\hat{s}_{\sigma}(t)=a(t)\left|G_{\sigma}\left[\omega_{i}(t)\right]\right| \cos \left\{\phi(t)+\angle G_{\sigma}\left[\omega_{i}(t)\right]\right\}$.

In the case of a monochromatic signal, i.e., a single cosine, the approximation (31) is exact; indeed, (31) may be regarded as a quasi-extension of the concept of the eigenfunctions of linear systems. The approximation (31) is also exact if $g_{\sigma}(t)$ is a unit impulse function. Otherwise, the error may be bounded according to the following result; the proof is supplied in Appendix A. First define,

$$
\begin{aligned}
\Delta_{p}\left(g_{\sigma}\right) & =\left[\int_{R} t^{2 \rho}\left|g_{\sigma}(t)\right|^{2} d t\right]^{1 / 2} \\
\delta(a) & =\left[\int_{R}|\dot{a}(t)|^{2} d t\right]^{1 / 2} .
\end{aligned}
$$

Theorem 1: Let $\varepsilon_{s}(t)=\left|s_{\sigma}(t)-\hat{s}_{\sigma}(t)\right|$, where $\hat{s}_{\sigma}(t)$ is given by (31). Then,

$$
\varepsilon_{s}(t) \leq \frac{4}{3} a_{\max } \Delta_{2}\left(g_{\sigma}\right) \cdot \delta\left(\omega_{i}\right)+2 \Delta_{1}\left(g_{\sigma}\right) \cdot \delta(a),
$$

where $a_{\max }=\sup _{t}|a(t)|$.

Thus, Theorem 1 bounds the error in terms of the concentration of $g_{\sigma}(t)$ in time (expressed as even moments of $\left|g_{\sigma}(t)\right|^{2}$ ), and the smoothness of the AM and FM functions $a(t)$ and $\omega_{i}(t)$ expressed as Sobolev 2-norms [4]. The bound (34) has another useful interpretation in the special case where $a(t)$ and $\omega_{i}(t)$ are bandlimited to the frequency intervals $\left[-\omega_{a}, \omega_{a}\right]$ and $\left[-\omega_{\phi}, \omega_{\phi}\right]$, respectively [8], [15], [16]. In this case $\delta(a) \leq\left|\omega_{a}\right| a_{\mathrm{rms}}$ and $\delta\left(\omega_{i}\right) \leq\left|\omega_{\phi}\right|\left(\omega_{i}\right)_{\mathrm{rms}}$, where $a_{\mathrm{ms}}=\left(\int a^{2}\right)^{1 / 2}$ and similarly for $\left(\omega_{i}\right)_{\mathrm{rms}}$.

Theorem 1 gives additional useful approximations for the derivatives of the response $s_{\sigma}(t)$

$$
\begin{aligned}
\frac{d^{k}}{d t^{k}} s_{\sigma}(t) \approx & a(t)\left|\omega_{i}(t)\right|^{k}\left|G_{\sigma}\left[\omega_{i}(t)\right]\right| \\
& \cdot \cos \left\{\phi(t)+\angle G_{\sigma}\left[\omega_{i}(t)\right]+k \frac{\pi}{2}\right\} .
\end{aligned}
$$

Here, (35) is assumed for $k=1,2,3$. The error in these approximations can be easily bounded by using Theorem 1 ; simply stated, the validity of (35) requires that the derivatives of $g_{\sigma}(t)$ of order up to 3 must be of short duration, in the sense that $\Delta_{m}\left(\dot{g}_{\sigma}\right), \Delta_{m}\left(\ddot{g}_{\sigma}\right), \Delta_{m}\left(\dddot{g}_{\sigma}\right)$ be small, for $m=1,2$ given by (32). 
Using reasoning similar to that in the development of Theorem 1, the following approximations for the energy $\Psi$ of the filtered signal component $s_{\sigma}(t)$ and its derivative will be essential

$$
\begin{aligned}
& \hat{\Psi}\left(s_{\sigma}\right)=a^{2}(t) \omega_{i}^{2}(t) \cdot\left|G_{\sigma}\left[\omega_{i}(t)\right]\right|^{2} . \\
& \hat{\Psi}\left(\dot{s}_{\sigma}\right)=a^{2}(t) \omega_{i}^{4}(t) \cdot\left|G_{\sigma}\left[\omega_{i}(t)\right]\right|^{2} .
\end{aligned}
$$

Note that (36) and (37) are exact for a monochromatic signal $s(t)=a_{0} \cos \left(\omega_{0} t\right)$, where $a_{0}, \omega_{0}$ are constant. A more general (nonmonochromatic) justification for these approximations is given next, in Theorem 2. First define,

$$
\bar{g}_{\sigma}=\int_{R}\left|g_{\sigma}(t)\right| d t
$$

Theorem 2: Let $\varepsilon_{\Psi}(t)=\left|\Psi\left(s_{\sigma}\right)-\hat{\Psi}\left(s_{\sigma}\right)\right|$, where $\hat{\Psi}\left(s_{\sigma}\right)$ is given by $(36)$. Then,

$$
\begin{aligned}
\mathcal{E}_{\Psi}(t) \leq & \frac{4}{3}\left(a_{\max }\right)^{2} \cdot\left[\bar{g}_{\sigma} \Delta_{2}\left(\ddot{g}_{\sigma}\right)+\bar{g}_{\sigma} \Delta_{2}\left(g_{\sigma}\right)\right. \\
& \left.+2 \bar{g}_{\sigma} \Delta_{2}\left(\dot{g}_{\sigma}\right)\right] \cdot \delta\left(\omega_{i}\right) \\
& +2 a_{\max } \cdot\left[\bar{g}_{\sigma} \Delta_{1}\left(\ddot{g}_{\sigma}\right)+\bar{g}_{\sigma} \Delta_{1}\left(g_{\sigma}\right)\right. \\
& \left.+2 \bar{g}_{\sigma} \Delta_{1}(\dot{g})\right] \cdot \delta(\alpha) .
\end{aligned}
$$

Theorem 2, while intuitive in view of Theorem 1, requires separate proof (given in Appendix B). However, both in Theorem 2 and the approximations (35)-(37), the error bounds are reduced by selecting $g_{\sigma}(t)$ to be of short duration and to have derivatives of up to order 2 of short duration. Slightly looser but intuitive bounds also apply when $a(t)$ and/or $\omega_{i}(t)$ are bandlimited (34).

\section{B. Filtered Noise Approximations}

Denote the autocorrelation of the filtered noise process $n_{\sigma}(t)$ in (30) by $R_{\sigma}(\tau)$ with associated power spectral density $\Phi_{\sigma}(\omega)=\left|G_{\sigma}(\omega)\right|^{2} \Phi(\omega)$. Of interest are the values that the $(2 k)$-th derivatives of $R_{\sigma}(\tau)$ take at the origin

$$
R_{o}^{(2 k)}(0)=\frac{1}{2 \pi} \int_{R}(j \omega)^{2 k}\left|G_{\sigma}(\omega)\right|^{2} \Phi(\omega) d \omega .
$$

An important, but nontrivial approximation will routinely be used

$$
R_{\sigma}^{(2 k)}(0)=\hat{R}_{\sigma}^{(2 k)}(\alpha),
$$

where for $\alpha \in R$ we define

$$
\hat{R}_{\sigma}^{(2 k)}(\alpha)=(-1)^{k} \alpha^{2 k}\left|G_{\sigma}(\alpha)\right|^{2} \Gamma_{\sigma}
$$

with

$$
\Gamma_{\sigma}=\frac{1}{2 \pi} \int_{R}\left|\frac{G_{\sigma}(\omega)}{G_{\sigma}\left(\omega_{c}\right)}\right|^{2} \Phi(\omega) d \omega
$$

the concentration of noise power within the passband of the filter $g_{\sigma}(t)$. The veracity of the approximation (41) requires some assumptions that are made clear in Theorem 3 (proved in Appendix $C$ ).

Theorem 3: Let $\mathcal{E}_{R_{\sigma}}^{(k)}(\alpha)=\left|R_{\sigma}^{(2 k)}(0)-\hat{R}_{\sigma}^{(2 k)}(\alpha)\right|$, where
$R_{\sigma}^{(2 k)}(0)$ and $\hat{R}_{\sigma}^{(2 k)}(\alpha)$ are given by $(41)-(43)$. Then,

$$
\begin{aligned}
\mathcal{E}_{R_{\sigma}}^{(k)}(\alpha)= & 2 \alpha^{2 k} \Phi_{\max }\left[2 \sqrt{2} k\left|\frac{\sigma}{\alpha}\right| V_{H}^{(1)}\left(\frac{\alpha-\omega_{c}}{\sigma}\right)\right. \\
& \cdot\left\{\left|\frac{\sigma}{\alpha}\right|^{2 k-1}\left[V_{H}^{(2 k-1)}\left(\frac{\omega_{c}}{\sigma}\right)\right]^{k}+1\right\} \\
& \left.+|1-| \frac{G_{\sigma}(\alpha)}{G_{\sigma}\left(\omega_{c}\right)} \mid\right]
\end{aligned}
$$

where $\Phi_{\max }=\sup _{\omega}|\Phi(\omega)|$ and $V_{H}^{(k)}$ is given by (26). $\square$

Careful examination of (44) reveals that, for arbitrary $\alpha$, the validity of the approximation (41) and (42) requires two important assumptions. First, the spread

$$
V_{H}^{(1)}\left(\frac{\alpha-\omega_{c}}{\sigma}\right)
$$

must be small; this occurs when $\alpha \approx \omega_{c}$ and when the bandwidth of $H(\omega)$ is small. Thus, the filter $G_{\sigma}(\omega)$ must be narrowband and $\alpha$ must fall near the center of the passband of $G_{\sigma}(\omega)$, i.e., near the filter center frequency $\omega_{c}$. Secondly, the quantity

$$
\left.|1-| \frac{G_{o}(\alpha)}{G_{\sigma}\left(\omega_{c}\right)}\right|^{2} \mid
$$

must be small; this requires either that $\alpha$ fall close to the filter center frequency $\omega_{c}$, or at least that $\alpha$ fall within the filter passband-if the in-band amplitude response $\left|G_{\sigma}(\omega)\right|$ is approximately flat. Fig. 2 illustrates these requirements on (45) and (46).

In the best case $\alpha=\omega_{c}$, the error bound (44) becomes

$$
\begin{aligned}
\varepsilon_{R_{\sigma}}^{(k)}\left(\omega_{c}\right) \leq & 4 \sqrt{2} k \omega_{c}^{2 k}\left|\frac{\sigma}{\omega_{c}}\right| \Phi_{\max } V_{H}^{(1)}(0) \\
& \cdot\left\{\left|\frac{\sigma}{\omega_{c}}\right|^{2 k-1}\left[V_{H}^{(2 k-1)}\left(\frac{\omega_{c}}{\sigma}\right)\right]^{k}+1\right\}
\end{aligned}
$$

which relative to the approximation $\hat{R}_{o}^{(2 k)}\left(\omega_{c}\right)$ given by (42) can be made constant across frequencies, for each value of $k$, by varying the bandwidth parameter $\sigma$ directly with the center frequency $\omega_{c}$. This observation motivates the constant $Q$, or wavelet scaling property of the multiband implementation described in Section V. Of course, small bandwidths will further reduce the approximation error, particularly when using (42) to approximate high-order derivatives of $R_{\sigma}(\tau)$ at $\tau=0$.

Specific forms of the approximation (41) and (42) will be of interest. In the sequel, whenever analyzing the filtered signal-plus-noise $f_{\sigma}(t)$ at time $t$, we will use (47) with $\alpha=\omega_{i}(t)$

$$
R_{\sigma}^{(2 k)}(0) \approx \hat{R}_{\sigma}^{(2 k)}\left[\omega_{i}(t)\right]
$$

This is a novel time-varying approximation to the autocorrelation of the filtered noise process. In making such an approximation, there is a tacit assumption that wherever $f_{\sigma}(t)$ is being analyzed, it is being done so with a 


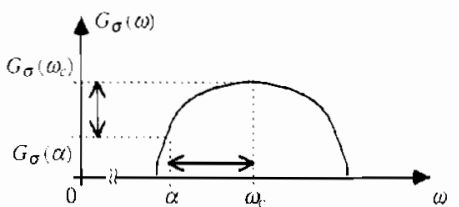

Fig. 2. The validity of the approximation (41) and (42) to the even derivatives $R_{\sigma}(\tau)$ at $\tau=0$ requires both that $\left|\alpha-\omega_{c}\right|$ be small and that $\mid G_{\sigma}(\alpha)$ - $G_{\sigma}\left(\omega_{c}\right) \mid$ be small. These requirements amount to specifying a narrow. flat passband for the filter $G_{\sigma}(\omega)$.

filter that is concentrated in the vicinity of the instantaneous signal frequency $\omega_{i}(t)$.

When analyzing filtered noise only, it will be convenient to use (47) with $\alpha=\omega_{c}$. This will be used to compare the responses of inactive channels (stimulated by noise only) with active channels (stimulated by signalplus-noise) in the multiband analysis of Section V.

It is desirable that the contribution of the noise element to the energy operator output $\Psi\left(f_{\sigma}\right)$ be minimized as much as possible. By using (48), approximations for the statistics of the energy of the filtered noise process can be obtained. From (10), (16), and (48):

$$
\begin{aligned}
& \mathrm{E}\left[\Psi\left(n_{\sigma}\right)\right]=-2 R_{\sigma}^{(2)}(0) \approx 2 \omega_{i}^{2}(t)\left|G_{\sigma}\left[\omega_{i}(t)\right]\right|^{2} \Gamma_{\sigma} \\
& \mathrm{E}\left[\Psi\left(\dot{n}_{\sigma}\right)\right]=2 R_{\sigma}^{(4)}(0) \approx 2 \omega_{i}^{4}(t)\left|G_{\sigma}\left[\omega_{i}(t)\right]\right|^{2} \Gamma_{\sigma} .
\end{aligned}
$$

Similarly, from (12), (17), and (48)

$$
\begin{aligned}
\operatorname{Var}\left[\Psi\left(n_{\sigma}\right)\right] & =3\left[R_{\sigma}^{(2)}(0)\right]^{2}+R_{\sigma}(0) R_{\sigma}^{(4)}(0) \\
& \approx 4 \omega_{i}^{4}(t)\left|G_{\sigma}\left[\omega_{i}(t)\right]\right|^{4} \Gamma_{\sigma}^{2} \\
\operatorname{Var}\left[\Psi\left(i_{\sigma}\right)\right] & =3\left[R_{\sigma}^{(4)}(0)\right]^{2}+R_{\sigma}^{(2)}(0) R_{\sigma}^{(6)}(0) \\
& \approx 4 \omega_{i}^{8}(t)\left|G_{\sigma}\left[\omega_{i}(t)\right]\right|^{4} \Gamma_{\sigma}^{2} .
\end{aligned}
$$

Clearly, both the means and the variances of $\Psi\left(n_{\sigma}\right)$ and $\Psi\left(\dot{n}_{\sigma}\right)$ are decreased by making $\Gamma_{\sigma}$ small, i.e., using a sufficiently narrow filter passband. But regardless of the bandwidth, from (49)-(52)

$$
\begin{aligned}
& \operatorname{Var}\left[\Psi\left(n_{\sigma}\right)\right] \approx \mathrm{E}^{2}\left[\Psi\left(n_{\sigma}\right)\right] \\
& \operatorname{Var}\left[\Psi\left(\dot{n}_{\sigma}\right)\right] \approx \mathrm{E}^{2}\left[\Psi\left(\dot{n}_{\sigma}\right)\right]
\end{aligned}
$$

which is important within the context of taking ratios of these types of quantities. In view of (53) and (54), it is not unlikely that $\Psi\left(n_{\sigma}\right)$ or $\Psi\left(i_{\sigma}\right)$ will take a value near zero, or a negative value. In the case of a low signal-tonoise ratio, computing the ESA subsequently becomes unreliable, as found in the next section; fortunately, the use of an appropriate filtering strategy can greatly improve the predicted results.

\section{Filtered Noisy AM-FM Signal Approximations}

First note that

$$
\Psi\left(f_{\sigma}\right)=\Psi\left(s_{\sigma}\right)+\Psi\left(n_{\sigma}\right)+2 \dot{s}_{\sigma} \dot{n}_{\sigma}-s_{\sigma} \ddot{n}_{\sigma}-\ddot{s}_{\sigma} n_{\sigma}
$$

the trailing terms of which are zero mean. Using (31), (35), (49), and (50), the expected values of the energy $\Psi$ of the filtered signal-plus-noise can be approximated:

$$
\begin{aligned}
& \mathrm{E}\left[\Psi\left(f_{\sigma}\right)\right] \approx \omega_{i}^{2}(t)\left|G_{\sigma}\left[\omega_{i}(t)\right]\right|^{2}\left[a^{2}(t)+2 \Gamma_{\sigma}\right] \\
& \mathrm{E}\left[\Psi\left(f_{\sigma}\right)\right] \approx \omega_{i}^{4}(t)\left|G_{\sigma}\left[\omega_{i}(t)\right]\right|^{2}\left[a^{2}(t)+2 \Gamma_{\sigma}\right] .
\end{aligned}
$$

It should be reemphasized that the validity of (55) and (56) utilizes the approximation (48) which requires the assumptions depicted in Fig. 2, i.e., that the instantaneous frequency $\omega_{i}(t)$ at time $t$ falls well within the passband of the filter with frequency response $G_{o}(\omega)$, and that the passband is nearly flat. Moreover, within the current analysis, without this assumption the signal component of the energy signals $\Psi\left(f_{\sigma}\right)$ and $\Psi\left(\dot{f}_{\sigma}\right)$ will also become negligible or vanish.

Although the ratio of (56) and (55) appears to be an appealing approximation of the expected value of the ESA (4), such an approximation must be carefully justified, particularly in view of (53) and (54). From (12), (17), (18), (20), (49)-(52),

$$
\operatorname{Var}\left[\Psi\left(f_{\sigma}\right)\right] \approx 4 \omega_{i}^{4}(t)\left|G_{\sigma}\left[\omega_{i}(t)\right]\right|^{4} \Gamma_{\sigma}\left[a^{2}(t)+\Gamma_{\sigma}\right]
$$

$$
\operatorname{Var}\left[\Psi\left(\dot{f}_{\sigma}\right)\right] \approx 4 \omega_{i}^{8}(t)\left|G\left[\omega_{i}(t)\right]\right|^{4} \Gamma_{\sigma}\left[a^{2}(t)+\Gamma_{\sigma}\right]
$$

$$
\operatorname{Cov}\left[\Psi\left(f_{\sigma}\right), \Psi\left(f_{\sigma}\right)\right] \approx 4 \omega_{i}^{6}(t)\left|G_{\sigma}\left[\omega_{i}(t)\right]\right|^{4} \Gamma_{\sigma}^{2}
$$

and

$$
\rho\left[\Psi\left(f_{\sigma}\right), \Psi\left(\dot{f}_{\sigma}\right)\right] \approx \Gamma_{\sigma} /\left[a^{2}(t)+\Gamma_{\sigma}\right]
$$

where $\rho$ denotes correlation coefficient.

We shall now reexamine the relative values of the expected values and variances of the energy signals, only this time following the filtering operation. Defining the instantaneous signal-to-noise ratio as a ratio of instantaneous signal power to average filtered noise power:

$$
\operatorname{SNR}_{\sigma}(t)=a^{2}(t) / \Gamma_{\sigma}
$$

we then find that

$$
\frac{\operatorname{Var}\left[\Psi\left(f_{\sigma}\right)\right]}{\mathrm{E}^{2}\left[\Psi\left(f_{\sigma}\right)\right]} \approx 4 \frac{\left[\operatorname{SNR}_{\sigma}(t)+1\right]}{\left[\operatorname{SNR}_{\sigma}(t)+2\right]^{2}} \approx \frac{\operatorname{Var}\left[\Psi\left(\dot{f}_{\sigma}\right)\right]}{\mathrm{E}^{2}\left[\Psi\left(\dot{f}_{\sigma}\right)\right]}
$$

and

$$
\frac{\operatorname{Cov}\left[\Psi\left(f_{\sigma}\right), \Psi\left(\dot{f}_{\sigma}\right)\right]}{\mathrm{E}\left[\Psi\left(f_{\sigma}\right)\right] \cdot \mathrm{E}\left[\Psi\left(\dot{f}_{\sigma}\right)\right]} \approx\left[\frac{2}{\operatorname{SNR}_{\sigma}(t)+2}\right]^{2} .
$$

In passing, we also note that

$$
\rho\left[\Psi\left(f_{\sigma}\right), \Psi\left(\dot{f}_{\sigma}\right)\right] \approx \frac{1}{\operatorname{SNR}_{\sigma}(t)+1} .
$$

Thus for sufficiently large SNR at time $t$, and if all assumptions regarding the filter are satisfied (underlying the key approximations (31), (35) and (48)), the energies $\Psi\left(f_{\sigma}\right)$ and $\Psi\left(\dot{f}_{\sigma}\right)$ are approximately uncorrelated; if the SNR at time $t$ is small, they have nearly a linear relationship. In view of (19) and (20), this is most accurate if the bandwidth of the system is sufficiently small. 
IV. Computing the ESA in the Presence of Noise

In this section, we justify the use of the ESA (4) and (5) in the presence of noise, where filtering is applied to reduce the noise contribution. Fig. 3 depicts the ESA system to be analyzed; compare with Fig. 1. Extensive use is made of the approximations for the output moments of the energy operator $\Psi$ developed in the preceding section. In addition, statistical justification of the combined filter/ ESA system is developed using approximations for the output moments of the ESA. As will be seen, it is difficult to develop statistical ESA approximations unless it is assumed that the signal-to-noise ratio is large.

\section{A. ESA Approximations for Large SNR}

Suppose that $\operatorname{SNR}_{\sigma}(t) \gg 1$ is sufficiently large that the ratios (62) and (63) are small; for example, using the value 0.1 to signify "small' requires [from (62)] that $\operatorname{SNR}_{a}(t)$ $>36.97$. Under this assumption and using (57)-(61), the following second-order approximations to the expectations of the ESA (4) and (5) are useful [11, p. 212]:

$$
\begin{aligned}
\mathrm{E}\left[\hat{\omega}_{i}^{2}(t)\right] & =\mathrm{E}\left[\frac{\Psi\left(\dot{f}_{\sigma}\right)}{\Psi\left(f_{\sigma}\right)}\right] \approx \frac{\mathrm{E}\left[\Psi\left(\dot{f}_{\sigma}\right)\right]}{\mathrm{E}\left[\Psi\left(f_{\sigma}\right)\right]}+\frac{\operatorname{Var}\left[\Psi\left(f_{\sigma}\right)\right] \cdot \mathrm{E}\left[\Psi\left(\dot{f}_{\sigma}\right)\right]-\operatorname{Cov}\left[\Psi\left(\dot{f}_{\sigma}\right), \Psi\left(f_{\sigma}\right)\right] \cdot \mathrm{E}\left[\Psi\left(f_{\sigma}\right)\right]}{\mathrm{E}^{3}\left[\Psi\left(f_{\sigma}\right)\right]} \\
& \approx \omega_{i}^{2}(t)\left\{1+\frac{4 \operatorname{SNR}_{\sigma}(t)}{\left[\mathrm{SNR}_{\sigma}(t)+2\right]^{2}}\right\} \approx \omega_{i}^{2}(t) \quad \text { (large SNR), }
\end{aligned}
$$

and

$$
\begin{aligned}
\mathrm{E}\left[\hat{a}^{2}(t)\right]= & \mathrm{E}\left[\frac{\Psi^{2}\left(f_{\sigma}\right)}{\Psi\left(\dot{f}_{\sigma}\right)}\right] \approx \frac{\mathrm{E}^{2}\left[\Psi\left(f_{\sigma}\right)\right]}{\mathrm{E}\left[\Psi\left(\dot{f}_{\sigma}\right)\right]} \\
& +\frac{\operatorname{Var}\left[\Psi\left(\dot{f}_{\sigma}\right)\right] \cdot \mathrm{E}^{2}\left[\Psi\left(f_{\sigma}\right)\right]-2 \operatorname{Cov}\left[\Psi\left(\dot{f}_{\sigma}\right), \Psi\left(f_{\sigma}\right)\right] \cdot \mathrm{E}\left[\Psi\left(f_{\sigma}\right)\right] \cdot \mathrm{E}\left[\Psi\left(\dot{f}_{\sigma}\right)\right]+\operatorname{Var}\left[\Psi\left(f_{\sigma}\right)\right] \cdot \mathrm{E}^{2}\left[\Psi\left(\dot{f}_{\sigma}\right)\right]}{\mathrm{E}^{3}\left[\Psi\left(\dot{f}_{\sigma}\right)\right]} \\
\approx & a^{2}(t)\left\{1+\frac{10 \mathrm{SNR}_{\sigma}(t)+4}{\operatorname{SNR}_{\sigma}(t)\left[\operatorname{SNR}_{\sigma}(t)+2\right]}\right\}\left|G_{\sigma}\left[\omega_{i}(t)\right]\right|^{2} \approx a^{2}(t)\left|G_{\sigma}\left[\omega_{i}(t)\right]\right|^{2} \quad \text { (large SNR). }
\end{aligned}
$$

Also for the second-order approximation for the variances of the ESA:

$$
\begin{aligned}
\operatorname{Var}\left[\hat{\omega}_{i}^{2}(t)\right] & =\operatorname{Var}\left[\frac{\Psi\left(\dot{f}_{\sigma}\right)}{\Psi\left(f_{\sigma}\right)}\right] \approx \frac{\operatorname{Var}\left[\Psi\left(f_{\sigma}\right)\right] \cdot E^{2}\left[\Psi\left(\dot{f}_{\sigma}\right)\right]-2 \operatorname{Cov}\left[\Psi\left(\dot{f}_{\sigma}\right), \Psi\left(f_{\sigma}\right)\right] \cdot \mathrm{E}\left[\Psi\left(f_{\sigma}\right)\right] \cdot \mathrm{E}\left[\Psi\left(\dot{f}_{\sigma}\right)\right]}{\mathrm{E}^{4}\left[\Psi\left(f_{\sigma}\right)\right]} \\
& \approx \omega_{i}^{4}(t) \frac{4\left[\mathrm{SNR}_{\sigma}(t)-1\right]}{\left[\operatorname{SNR}_{\sigma}(t)+2\right]^{2}}
\end{aligned}
$$

and

$$
\begin{aligned}
\operatorname{Var}\left[\hat{a}^{2}(t)\right]= & \operatorname{Var}\left[\frac{\Psi^{2}\left(f_{\sigma}\right)}{\Psi\left(\dot{f}_{\sigma}\right)}\right] \approx \frac{\mathrm{E}^{2}\left[\Psi\left(f_{\sigma}\right)\right]}{\mathrm{E}^{4}\left[\Psi\left(\dot{f}_{\sigma}\right)\right]}\left\{\operatorname{Var}\left[\Psi\left(\dot{f}_{\sigma}\right)\right] \cdot \mathrm{E}^{2}\left[\Psi\left(f_{\sigma}\right)\right]-4 \operatorname{Cov}\left[\Psi\left(f_{\sigma}\right), \Psi\left(f_{\sigma}\right)\right]\right. \\
& \left.\cdot \mathrm{E}\left[\Psi\left(f_{\sigma}\right)\right] \cdot \mathrm{E}\left[\Psi\left(\dot{f}_{\sigma}\right)\right]+4 \operatorname{Var}\left[\Psi\left(f_{\sigma}\right)\right] \cdot \mathrm{E}^{2}\left[\Psi\left(\dot{f}_{\sigma}\right)\right]\right\} \approx 4 a^{4}(t) \frac{5 \operatorname{SNR}_{\sigma}(t)+1}{\operatorname{SNR}_{\sigma}^{2}(t)}\left|G_{\sigma}\left[\omega_{i}(t)\right]\right|^{4}
\end{aligned}
$$

At first glance it may appear that the variances (66) and (67) of the ESA (4) and (5) increase dramatically with the fourth powers of the AM and FM functions $\omega_{i}(t)$ and $a(t)$. both of which become negligible at reasonably high values of $\operatorname{SNR}_{\sigma}(t)$. Fig. 4 plots (68) and (69) versus $\operatorname{SNR}_{\sigma}(t)$ $>10$. As is apparent from the plots, both ratios fall off 


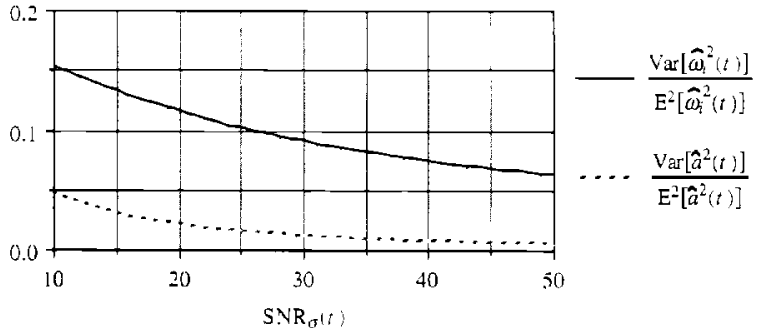

Fig. 4. Plots of the ratios (68) and (69). For small values of the plotted functions, the approximations (70) and (71) may be considered valid.

fairly rapidly; (68) falls to 0.1 at $\operatorname{SNR}_{\sigma}(t)=26(28 \mathrm{db})$, whereas $(69)$ falls to 0.1 at $\operatorname{SNR}_{\sigma}(t) \approx 34(31 \mathrm{db})$.

It subsequently follows from (64)-(69) that for $\operatorname{SNR}_{\sigma}(t)$ sufficiently large we may confidently assume that

$$
\begin{aligned}
& \hat{\omega}_{i}^{2}(t) \approx \omega_{i}^{2}(t) \\
& \hat{a}^{2}(t) \approx a^{2}(t)\left|G_{\sigma}\left[\omega_{i}(t)\right]\right|^{2} .
\end{aligned}
$$

If $(70)$ is valid, then in $(71), \omega_{i}(t)$ may be estimated first using (5) and used to compute $G_{\mathrm{o}}\left[\omega_{i}(t)\right]$. Another simple approach is to use filters with approximately flat in-band responses, so that

$$
\left|G \sigma\left[\omega_{i}(t)\right]\right| \approx\left|G_{0}\left(\omega_{c}\right)\right| .
$$

Of course this is just another statement of the constraint (46), which is important in making the key approximation (48) accurate, and is used in the simulations given in Section VI.

\section{Multiband Filtering and ESA}

Equations (31) and (35) imply that unless the instantaneous frequency $\omega_{i}(t)$ falls within the passband of the filter $G_{\sigma}(\omega)$, the energy signals $\Psi\left(s_{\sigma}\right)$ and $\Psi\left(\dot{s}_{\sigma}\right)$ will become negligible or vanish. Therefore, by the selection of an appropriate filter (passband) at time $t$, it becomes possible to isolate an AM-FM signal component and to compute its energy $\Psi$. Moreover, it becomes possible to reject noise components not falling within the vicinity of the desired local AM-FM component.

These very useful observations about locally coherent signals (signals with a strong local modulation structure) can be regarded as extending the well-known global properties of filtered sinusoidal signals immersed in noise. With it comes a caveat: in order to isolate the local modulation energy of an AM-FM signal component of the form (1) in the presence of noise, it becomes necessary to establish a filter passband in advance, either by estimating the local center frequency by a tracking procedure, or by utilizing a bank of bandpass filters that sample the frequency domain sufficiently densely. This is particularly important since the instantaneous signal frequencies may sweep the signal spectrum, implying that multiple filters will be involved, at different times, in capturing the modulation signal $s(t)$. Here, the second approach is explored. In particular, criteria for channel filter selection and for the design of a multiband sampling of the fre- quency domain are discussed in depth. Significantly, it is found that uniform (worst-case) performance across frequency bands is naturally ensured through the use of a constant- $Q$ or wavelet-like scaling of the channel filters.

Fig. 5 depicts a block diagram of the multiband energy operator system that is proposed and analyzed in detail in this section. The signal $f(t)$ is divided into multiple passbands using $M$ filters having impulse responses $g_{m}(t)$, frequency responses $G_{m}(\omega)$, with associated center frequencies $\omega_{m}$ and bandwidth parameters $\sigma_{m}$, producing outputs $f_{m}(t) ; m=1, \cdots, M$. The filters $G_{m}(\omega)$ are all assumed to have the form (22)-(29). Section V-A discusses the design of the individual channel filters $G_{m}(\omega)$, based on the observations made in the preceding sections, while Section V-B discusses the multiband implementation. Following filtering, the process of energy demodulation using the operator $\Psi$ is applied to each output. Although not made explicit in Fig. 5, at each instant $t$ the response having the maximum normalized energy

$$
\Psi *(t)=\max _{1 \leq m \leq M} \frac{\Psi\left[f_{m}(t)\right]}{\left|G_{m}\left(\omega_{m}\right)\right|^{2}}
$$

is used as the analysis signal, i.e., it is input to the ESA. In this way, a filter is made available with large response to the signal component $s(t)$, by ensuring that the instantaneous frequency $\omega_{i}(t)$ falls within one of the filter subbands motivating the multiband implementations described in Section V-B. The efficacy of the operation (72) is examined in Section V-C. Thus, a filter $g_{\sigma}$ containing the instantaneous frequency within its passband will likely be used in computing the ESA, yielding a stable, noiseresistant result. In this case, depicted by the dotted lines in Fig. 5, the behavior of the ESA is well described by the results of the previous sections. Section V-C studies the strategy of utilizing the channel with maximum energy output $\Psi^{*}(t)$ as the analysis channel. A similar strategy was employed using Gabor wavelets in a digital image analysis application in [4], although the actual filter responses, rather than computed energies, were used to determine the analyzing channels.

The strategy depicted in Fig. 5 presents a novel and interesting approach to isolating an AM-FM signal that may be modified. The approach used here yields the channel having the maximum product of channel response and Teager energy. This means that the magnitude of the instantaneous frequency $\left|\omega_{i}(t)\right|$ is deemed equally important as the amplitude function $|a(t)|$ in detecting the signal, which, while not usual, does have application. However, changing (72) to agree with more conventional criteria is straightforward. For example, the ESA may be computed for each channel, and the analysis channel selected on the basis of the maximum estimated $\left|\omega_{i}(t)\right|$ (if it is desired to find high-frequency AM-FM functions), or on the maximum estimated $|a(t)|$ (if it is desired to find high-amplitude AM-FM functions, which is the most likely goal). A simple approach which well approximates the latter strategy is to modify the criteria (72) by normalizing also by 


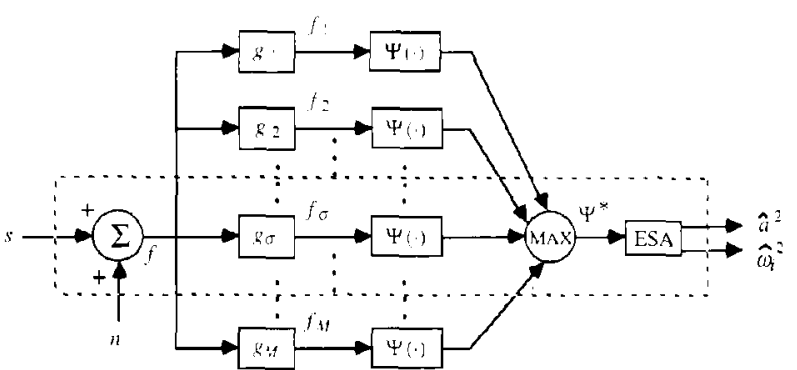

Fig. 5. Block diagram depicting multiband filtering and energy separation of a noisy AM-FM signal. The dotted lines indicate a single subsystem as analyzed in the preceding sections, which may be regarded as having the largest energy reponse $\Psi^{*}$ at time $t$

the squared channel center frequency:

$$
\Psi^{*}(t)=\max _{1 \leq m \leq M} \frac{\left.\Psi \mid f_{m}(t)\right]}{\left|\omega_{m} G_{m}\left(\omega_{m}\right)\right|^{2}} .
$$

Whichever method is used, the multiband strategy still affords superior predicted and demonstrated performance. In this paper, (72) will continue to be used for simplicity, and also novelty, of exposition.

\section{A. Filter Design}

Before exploring the design of the multiband implementation, criteria for selecting the individual filters comprising the signal decomposition are explored (with the multiband implementation in mind). There are a variety of criteria, some of them conflicting, that affect the design of the individual filters, or more precisely, the design of the low-pass equivalent filter $H(\omega)$ in (22)-(24). The architecture of the multi-band implementation will be dictated by a set of frequency translated and dilated versions of the bandpass filter $G_{\sigma}(\omega)$ defined by (22) and (23).

As will be seen, both low-uncertainty filters [4] and filters with a flat in-band responses have certain advantages. Indeed, Theorems 1 and 2 and (35) suggest that, in order that the modeling of the deterministic problem be accurate, the channel filters be selected such that the temporal energy durations

$$
\Delta_{p, k}\left(g_{\sigma}\right)=\Delta_{p}\left[\frac{d^{k}}{d t^{k}} g_{\sigma}\right], \quad k=0,1,2,3
$$

be made as small as possible, for $p=1,2$, where $\Delta_{p}(\cdot)$ is given by (32). It is enough to consider $p=1$. It is not difficult to state this in a simple way as constraints on the low-pass equivalent filters $h_{\sigma}$. With $g_{\sigma}$ and $h_{\sigma}$ related by (22), it is a simple matter to show that $\Delta_{l}\left(g_{\sigma}\right) \leq \Delta_{1}\left(h_{\sigma}\right)$, and that further, for each $k=0,1,2.3$, there exists constants $b_{k, i} ; i=0, \cdots, k$, such that

$$
\Delta_{i, k}\left(g_{\sigma}\right) \leq \sum_{i=0}^{k} b_{k, i} \Delta_{l, k}\left(h_{\sigma}\right) .
$$

Thus, it suffices to take the temporal energy durations of $h_{\sigma}$ and its low-order derivatives to be small. However, by the arguments posed by Theorem 3 , in order that the mod- eling of the statistical problem be accurate, the spectral energy duration $V_{H}^{(1)}(0)$ given by (27) should also be taken small. Ignoring momentarily constraints on the derivatives of $h_{0}$, simultaneously optimizing these criteria, by for example, minimizing the product

$$
\Delta_{1}(h) V_{H}^{(1)}(0)
$$

inevitably leads to the minimum uncertainty filters of the form [4]

$$
\begin{aligned}
h_{\sigma}(t) & =(2 \pi)^{-1 / 4} \sqrt{\sigma} \exp \left\{-(\sigma t)^{2} / 4\right\} \\
H_{\sigma}(\omega) & =(2 \pi)^{1 / 4} \sqrt{\frac{2}{\sigma}} \exp \left\{-\left(\frac{\omega}{\sigma}\right)^{2}\right\}
\end{aligned}
$$

which are unit-energy Gaussian functions. The functions (22), which are frequency-shifted Gaussians, are then the real-valued Gabor functions. A couple of points need to be considered before taking these as one possible basis for a multi-band implementation. First, in (29) it was assumed (for simplicity of exposition) that the shifted filters $H_{\sigma}\left(\omega \pm \omega_{c}\right)$ not have any spectral overlap; clearly, the functions (74), which have infinite support, violate this criteria. However, by taking the filter bandwidths sufficiently narrow, say one octave, the overlap will be extremely small [4]. In any practical implementation, the filter bandwidths will be effectively limited to achieve (29). Of course, there exist other low-uncertainty filters that have strictly finite support, such as prolate functions [15], which could be used. The difference in performance, however, would likely be microscopic. Finally, in defining low uncertainty filters that minimize the product (73) the durations $\Delta_{1, k}\left(h_{\sigma}\right)$ of the derivatives have been ignored. However, the low-order derivatives of Gaussians also have good time/frequency localization properties [17].

There is another important criterion to be considered. Theorem 3 suggests the constraint (46), which implies that when $\alpha \approx \omega_{c}$, it is desirable that $G_{\sigma}(\alpha) \approx G_{\sigma}\left(\omega_{c}\right)$. In other words, the passband of $H(\omega)$ should be approximately flat. Of course, this criterion conflicts with the choice of a Gaussian/Gabor configuration, since the Gaussian frequency characteristic rolls off quickly within the passband, and slowly at the transition, rather than having an abrupt transition from a flat passband. While it is an interesting problem to consider the design of filters that are both low uncertainty and also have relatively flat passbands, there is no immediately apparent procedure for such a design. In any case, the error bounds described in the preceding represent worst-case performances. Therefore, simple multiband filter configurations are considered next, consisting either of Gabor functions or ideal (flat) bandpass filters. In either case the use of a multiscale, dyadic wavelet-like filter bank configuration is motivated.

\section{B. Multiband Design}

Once a basic low-pass filter $H(\omega)$, or basic bandpass filter $G_{\sigma}(\omega)$ has been selected, the next step is the choice 
of a tesselation of the frequency axis by a set of multiple frequency translations and dilations of $G_{\sigma}(\omega)$. Since in this development, signals are being analyzed on continuous domains, the practical assumption is made that the system of interest produces signals that fall within a specific (possibly wide) band of frequencies; of course, any real-world system approximates this hypothesis. Thus, assume the overall bandwidth of the analysis system to be fixed at an upper limit $\Omega_{c}$, i.e., the magnitude $|S(\omega)|$ of the Fourier integral $s(t)$ is guaranteed to be negligible for all $|\omega|>$ $\Omega_{c}$.

Many arguments have been put forth for the use of time/ frequency signal analysis windows having a constant width (localization) on a logarithmic scale [18]-[21]. One of the simplest, yet most compelling observations, applies to the local analysis of monochromatic functions; lower frequencies require larger analysis windows in order to capture the signal's period. Since we are dealing with signals that may be termed "locally quasimonochromatic," similar arguments apply. However, within the current modeling framework, the bound in Theorem 3 supplies additional motivation, as discussed next.

The important approximations developed for the local noise response in the presence of an AM-FM signal, all of which are based on (48), can be improved by designing the basic filter $H(\omega)$ according to certain prescriptions, as in Section V-A. However, the modeling error bound (47) is also scaled by

$$
\left|\frac{\sigma}{\omega_{c}}\right|^{2 k-1}\left[V_{H}^{(2 k-1)}\left(\frac{\omega_{c}}{\sigma}\right)\right]^{k}
$$

so it is necessary that the channel bandwidths be small, but more importantly, in order to maintain consistent (worst-case) predicted perforamnce across the filter channels the error bound can be made constant across the spectrum by taking the bandwidth (dilation) parameter $\sigma_{m}$ for each channel $G_{m}(\omega)$ to be the inverse (up to a multiplicative constant) of the center frequency (translation) parameter $\omega_{m}$ :

$$
\frac{\sigma_{m}}{\omega_{m}}=\text { constant; } \quad m=1, \cdots, M-1
$$

which implies a constant logarithmic spacing between the filters. In other words, constant- $Q$ (wavelet scaled) filter banks will provide uniform worst-cast performance across channels.

Certainly, uniform (worst-case) performance across channels is a design criterion that may be modified. If it is desired to achieve improved performance over some sub-band of frequencies, then this can be accomplished by narrowing the filters bandwidths (using more filters) over that frequency range. Nevertheless, it is significant that the constant- $Q$ property falls naturally out of the analysis. Given the specific goal of estimating $a(t)$ and $\omega_{i}(t)$, uniform performance for small or large values of $\omega_{i}(t)$ is obviously desirable unless there is a specific reason otherwise. In any case, the (worst-case) performance from each channel will depend directly on its $Q$.
Thus, regardless of the exact filter specification, define the set of $M$ center frequencies for the subband filters (notice the indexing from higher frequencies to lower frequencies) by a dyadic relation, yielding one-octave separation:

$$
\omega_{m}=3 \cdot 2^{-(m+1)} \Omega_{c} ; \quad m=1, \cdots, M-1 .
$$

The baseband filter will be taken to be an unshifted (lowpass) filter:

$$
G_{M}(\omega)=\frac{1}{j \sqrt{\sigma_{M}}} H\left(\frac{\omega}{\sigma_{M}}\right)
$$

while the subband channel filters are given by

$$
\begin{gathered}
G_{m}(\omega)=\frac{1}{j \sqrt{\sigma_{m}}}\left[H\left(\frac{\omega-\omega_{m}}{\sigma_{m}}\right)-H\left(\frac{\omega+\omega_{m}}{\sigma_{m}}\right)\right] \\
m=1, \cdots, M-1
\end{gathered}
$$

Gabor Wavelets: The real-valued (sine) Gabor wavelets defined by (22), (28), (74) and (75) are of the form

$$
\begin{aligned}
G_{m}(\omega)= & (2 \pi)^{-1 / 4} \frac{1}{j} \sqrt{\frac{2}{\sigma_{m}}}\left[\exp \left\{-\left(\frac{\omega-\omega_{m}}{\sigma_{m}}\right)^{2}\right\}\right. \\
& \left.-\exp \left\{-\left(\frac{\omega+\omega_{m}}{\sigma_{m}}\right)^{2}\right\}\right] .
\end{aligned}
$$

The bandpass functions (78) satisfy the usual wavelet admissibility conditions [18]-[21]: complete orthonormal decomposition of any signal $f(t) \in L^{2}(\boldsymbol{R})$ can be constructed using a basis consisting of frequency translates and dilates of (78), from which the signal may be exactly reconstructed, in principle. However, from a practical perspective, in the application being considered here, where highly specific signal components (AM-FM signals) are being extracted in the presence of significant noise, it is most important that the frequency domain be adequately sampled by the filter set, rather than ensuring the perfect reconstruction property of the filter responses.

Nevertheless, if the filter tesselation is defined such that the filters intersect at half-peak, then both criteria are satisfied. One-octave (half-peak bandwidth) Gabor filters achieve this prescription exactly. In this case the filter bandwidth parameters satisfy

$$
\sigma_{m}=\frac{\omega_{m}}{3 \sqrt{\ln 2}}=\frac{2^{-(m+1)}}{\sqrt{\ln 2}} \Omega_{c} ; \quad m=1, \cdots, M-1 .
$$

We choose $\sigma_{M}=2 \sigma_{M-1}$ for the baseband filter, in order that the filters $G_{M-1}(\omega), G_{M}(\omega)$ intersect at the half-peak responses. Fig. 6 depicts a decomposition of the unit frequency interval $[0,1]$ (i.e., $\Omega_{c}=1$ ) using five Gabor wavelets defined by (75), (77)-(79).

Littlewood-Paley Wavelets: Littlewood-Paley wavelets 


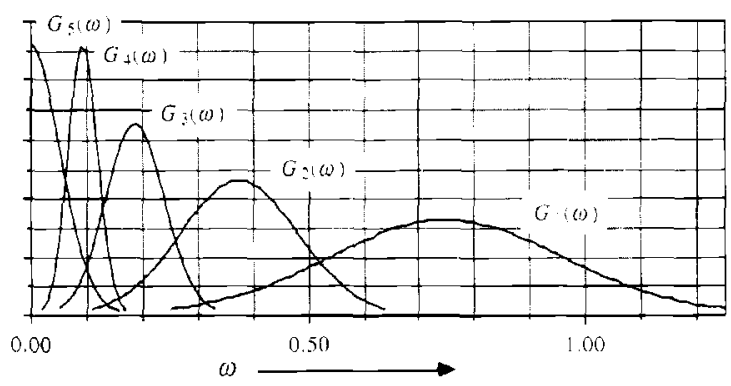

Fig. 6. Multiband Gabor wavelet filter decomposition of the unit frequency interval $[0.1]$ into five subbands of constant (unity) octave bandwidth (except the baseband).

$[21$, p. 105] satisfying (28) have the form

$$
\begin{aligned}
G_{m}(\omega)= & \frac{1}{j} \sqrt{\frac{\pi}{\sigma_{m}}}\left[I_{[-1,1]}\left(\frac{\omega-\omega_{m}}{\sigma_{m}}\right)\right. \\
& \left.-I_{[-1,1]}\left(\frac{\omega+\omega_{m}}{\sigma_{m}}\right)\right]
\end{aligned}
$$

where $I_{[-1,1]}$ is the indicator function of the interval [ -1 , $1]$. Thus ( 80 ) represents the ideal case of maximally flat filters also having good spectral localization, but ripple in the time domain. Although not realizable in practice, the bandpass functions (80) also satisfy the usual wavelet admissibility conditions [18]-[21]. Here the prescriptions for perfect reconstruction and complete coverage of the system bandwidth are in agreement. Thus, defining center frequencies $\omega_{m}$ according to (75), (76) and the bandwidth parameters according to

$$
\sigma_{m}=\frac{\omega_{m}}{3}=2^{-m+1} \Omega_{i} ; \quad m=1, \cdots, M-1
$$

and $\sigma_{M}=2 \sigma_{M-1}$ (baseband filter) once again yields a set of unity-octave filters with unity octave spacing. The (positive frequency) filter passbands are the intervals $\left[2^{-m} \Omega_{c}, 2^{-m+1} \Omega_{c}\right], m=1, \cdots . M-1$. Fig. 7 depicts a decomposition of the unit frequency interval $[0,1]$ using five Littlewood-Paley wavelets defined by (75), (77), (80), and (81). Of course, modelling the bandpass filters as ideal is an analytic convenience. In practive, the filter characteristics may be approximated using, e.g., quadrature mirror filters [22], related subband techniques [23], or standard bandpass filter design procedures [26].

Comments: Of course, the use of one-octave filters with one-octave separations is somewhat arbitrary in the presence of significant noise, where the signal-to-noise ratio may be low, it may be advisable to use a large number of filters having narrow bandwidths, such as $(1 / 2)$-octave filters with $(1 / 2)$-octave separations.

\section{Channel Selection by Maximum Energy Response}

In this subsection the technique (72) of selecting an analysis band for the extraction of signal frequency modulations by finding the channel having the largest energy response is studied. Suppose that the signal is given by

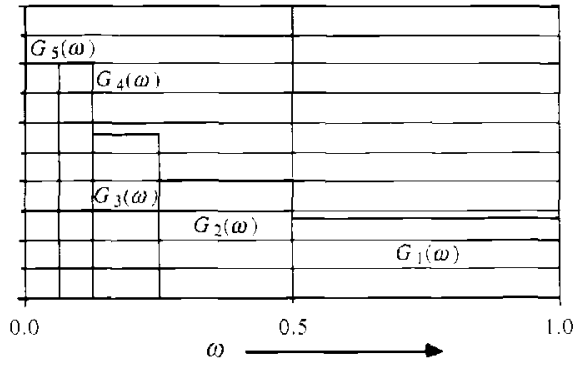

Fig. 7. Multiband filter decomposition of the unit frequency interval [0, 1] using five ideal subbands of constant (unity) octave bandwidth (except the baseband)

(1) and assume that the instantaneous frequency $\omega_{i}(t)$ falls within the passband of the filter $G_{m}$, where for simplicity $m \neq M$. Then from (55), and defining $\operatorname{SNR}_{m}(t)$ using (61) with $\sigma=\sigma_{m}$, the normalized expected signal-plus-noise energy is approximately

$$
\mathrm{E}\left[\Psi\left(f_{m}\right)\right] \approx a^{2}(t) \omega_{i}^{2}(t)\left|\frac{G_{m}\left[\omega_{i}(t)\right]}{G_{m}\left(\omega_{m}\right)}\right|^{2}\left[1+\frac{2}{\operatorname{SNR}_{m}(t)}\right]
$$

For every other channel $G_{q}, q \neq m$, from (36) and (10), and using (41) with $\alpha=\omega_{q}$, the normalized expected signal-plus-noise energy is approximately

$$
\mathrm{E}\left[\Psi\left(f_{q}\right)\right]=a^{2}(t) \omega_{i}^{2}(t) \cdot\left|\frac{G_{q}\left[\omega_{i}(t)\right]}{G_{q}\left(\omega_{q}\right)}\right|^{2}+2 \omega_{q}^{2} \Gamma_{q} .
$$

with $\Gamma_{q}$ given by (43). Clearly, in the absence of noise

$$
\mathrm{E}\left[\Psi\left(f_{m}\right)\right] \gg \mathrm{E}\left[\Psi\left(f_{q}\right)\right]
$$

if the filters are properly designed. Otherwise the ratio of expected energies:

$$
\begin{aligned}
\frac{\mathrm{E}\left[\Psi\left(f_{q}\right)\right]}{\mathrm{E}\left[\Psi\left(f_{m}\right)\right]} \approx & {\left[\frac{\mathrm{SNR}_{m}(t)}{\mathrm{SNR}_{m}(t)+2}\right] \cdot\left|\frac{G_{m}\left(\omega_{m}\right)}{G_{m}\left[\omega_{i}(t)\right]}\right|^{2} } \\
& \cdot\left\{\left|\frac{G_{q}\left[\omega_{i}(t)\right]}{G_{q}\left(\omega_{q}\right)}\right|^{2}+\frac{2}{\operatorname{SNR}_{q}(t)} \cdot\left(\frac{\omega_{q}}{\omega_{i}(t)}\right)^{2}\right\} .
\end{aligned}
$$

The first term inside the brackets is clearly negligible. The second term inside the brackets will be small if the signalto-noise ratio $\mathrm{SNR}_{q}(t)$ is large. Hence, it is important that the bandwidth of each channel filter be taken snall to achieve large signal-to-noise ratios across all of the channels, in order that the strategy of using the channel with the maximum normalized response (72) as the analysis band will be most effective. However, the scaling by the factor $\omega_{q}^{2} / \omega_{i}^{2}(t)$ does indicate that the ESA will be sensitive when it is desired to detect low-frequency AM-FM signals immersed in high-frequency noise. This is because of the strategy (72) that is used. As discussed below (72), it is possible to ameliorate this effect by computing the ESA for every channel.

The only other question concerns the behavior of the overall ESA as the instantaneous frequency $\omega_{i}(t)$ sweeps across channels. Conceptually, there is a discontinuity in the flow of the algorithm when there is a transition from 
one maximizing channel to another according to (72). In view of the above discussion this occurs when the instantaneous frequency makes a transition from one passband to another. However, under rather general conditions, the ESA (4) and (5) gives continuously-varying results.

In applying the energy operator $\Psi$ to the signal (1) as in (3), it must be assumed that the signal $s(t)$ is a twicedifferentiable function. However, if the signal is filtered first, then it is more convenient to impose additional conditions on the filter impulse response such that the ESA will be regular. Indeed, it is sufficient to assume that the low-pass equivalent function $h(t)$ is continuously threetimes-differentiable, a condition clearly met by the example wavelet configurations discussed in Section V-B. Then, the energy responses $\Psi\left[f_{m}(t)\right] ; m=1, \cdots, M$ will all be continuous functions [see (B.6)], as will be the associated energies $\Psi\left[\dot{f}_{m}(t)\right]$ necessary for the ESA computations. Since the maxima of continuous functions is continuous, the maximum Teager response $\Psi^{*}(t)$ will also be continuous. Accordingly, the associated energy $\Psi^{*}[\dot{f}(t)]$ should also be continuous. In order to assure this, the energy-of-derivatives could also be computed for every channel and the maximum taken, although according to the approximation (56) this is likely unnecessary in practice. Finally, since the ESA (4) and (5) is defined in terms of continuous mappings, the ESA responses shown in Fig. 5 will be continuous functions.

Comments: The above analysis could, in principle, be extended in a natural and interesting way by casting the channel selection problem, currently implemented via (72), as an $M$-ary hypothesis testing problem. Such an approach would lead to a probabilistic description of the overall multiband ESA performance as a function of the noise, the filter bandwidths, and the number of filters. Complicating this approach, however, is the necessity of expressing the necessary output conditional distributions of the Teager-Kaiser operator for a signal-plus-noise input. As indicated in Section II, this problem is complicated even for the case of zero signal; for the case of signal-plus-noise, it is much more difficult (Section II-C). It is for this reason that the stochastic analysis in this paper has been restricted to the computation of useful moments of the energy operator output.

\section{Examples and Simulations}

In this section, examples are provided of the predicted ESA performance for a sinusoidal signal (Section VI-A) and for a chirp signal (Section VI-B). Section VI-C provides actual MATLAB simulation results, using an approximate implementation of the Littlewood-Paley filter bank described in Section V-B.

\section{A. Sinusoidal Signal-Monochromatic Noise}

As an instructive example consider the case of a pure sinusoidal signal

$$
s(t)=a_{0} \cos \left(\omega_{0} t\right)
$$

where the signal frequency falls in an assumed frequency interval: $\omega_{0} \in[0,1]$ (i.e., $\Omega_{c}=1$ for simplicity). Further assume that the signal is embedded in zero-mean, additive Gaussian monochromatic noise $n(t)$ with autocorrelation

$$
R(\tau)=A^{2} \cos \left(\omega_{1} \tau\right)
$$

We shall now study the efficacy of the overall system depicted in Fig. 5, using the Littlewood-Paley wavelet configuration (80). This example (82) and (83) is canonical, since nearly all of the approximations [except (64)-(67)] made prior to this point hold exactly, provided that $\omega_{0}$ and $\omega_{1}$ fall into different frequency bands, although that case will be considered as well. Of course, this example represents the simplest possible case, where there is no information in the AM-FM signals. It is an important application, since it is often of interest to capture a dominant sinusoidal signal in the presence of other random periodic signals(s). The example also provides insights into the general performance of the ESA in the presence of filtered noise; for signals having slowly-varying modulating functions, this example is largely applicable. Of course, if either or both of the signal amplitude and the signal phase vary with extreme rapidity, then the approximations, particularly those developed in Theorems 1 and 2, are not guaranteed to be close. One interpretation of this, however, is that it is simply quite difficult to track a signal with rapidly fluctuating AM or FM information.

Assume that the signal frequency $\omega_{0}>0$ falls within the passband of the filter $G_{m}(\omega)$, and that the noise frequency $\omega_{1}>0$ falls out of band, viz., in the band of the filter $G_{q}(\omega)$, where for simplicity $m, q \neq M$. The case where the signal and noise frequencies fall into the same band is really the same as the case where no bandpass prefiltering is applied; that is considered next. From (36), (37), (80), and (81) the exact normalized in-band filtered signal energies are

$$
\begin{aligned}
& \frac{\Psi\left(f_{m}\right)}{\left|G_{m}\left(\omega_{m}\right)\right|^{2}}=a_{0}^{2} \omega_{0}^{2} \\
& \frac{\Psi\left(f_{m}\right)}{\left|G_{m}\left(\omega_{m}\right)\right|^{2}}=a_{0}^{2} \omega_{0}^{4}
\end{aligned}
$$

since the noise falls out-of-band.

For the out-of-band energies, the filter/energy operator system responds to the noise only. The exact normalized energy moments are found by directly evaluating (40) in (49)-(52), (80), and (81)

$$
\frac{\mathrm{E}\left[\Psi\left(n_{q}\right)\right]}{\left|G_{q}\left(\omega_{q}\right)\right|^{2}}=2 A^{2} \omega_{1}^{2} .
$$

The responses of all other channels will be zero. The ratio of $(84)$ to $(85)$ :

$$
\left(\frac{1}{2}\right)\left(\frac{a_{0} \omega_{0}}{A \omega_{1}}\right)^{2}
$$

makes it clear that for sufficiently large signal-to-noise ratio $a_{0}^{2} \omega_{0}^{2}>2 A^{2} \omega_{1}^{2}$, the correct channel $G_{m}(\omega)$ will pro- 
vide the largest energy response, resulting in an exact ESA computation (4) and (5). Otherwise the approach will clearly fail, corresponding to the case where there exists a periodic noise signal burying the signal of interest. However, the main point to be made is that, once the correct channel is selected, the ESA performs extremely well. Comparison-Without Filtering: It is interesting to compare the performance of the energy operator in the preceding with the case where the energy operator and ESA computations are not preceded by bandpass filtering. In this case, the exact expectations of the signal energies are

$$
\begin{aligned}
& \mathrm{E}[\Psi(f)]=a_{0}^{2} \omega_{0}^{2}+2 A^{2} \omega_{1}^{2} \\
& \mathrm{E}[\Psi(f)]=a_{0}^{2} \omega_{0}^{4}+2 A^{2} \omega_{1}^{4}
\end{aligned}
$$

which is a weighted linear combination of even powers of the signal $\left(\omega_{0}\right)$ and noise $\left(\omega_{1}\right)$ frequencies. Hence, even in the case of a fairly large signal-to-noise ratio, the ESA computations will be inaccurate, since, in the best case [from (64) and (65)]

$$
\begin{aligned}
& \mathrm{E}\left[\hat{\omega}_{i}^{2}(t)\right] \approx \frac{a_{0}^{2} \omega_{0}^{4}+2 A^{2} \omega_{1}^{4}}{a_{0}^{2} \omega_{0}^{2}+2 A^{2} \omega_{1}^{2}} \\
& \mathrm{E}\left[\hat{a}^{2}(t)\right] \approx \frac{\left(a_{0}^{2} \omega_{0}^{2}+2 A^{2} \omega_{1}^{2}\right)^{2}}{a_{0}^{2} \omega_{0}^{4}+2 A^{2} \omega_{1}^{4}} ;
\end{aligned}
$$

neither resembles the correct values $a_{0}^{2}$, $\omega_{0}^{2}$ unless $a_{0}^{2} \omega_{0}^{2}$ $>2 A^{2} \omega_{1}^{2}$ or $a_{0}^{2} \omega_{0}^{2} \approx 2 A^{2} \omega_{1}^{2}$.

\section{B. Chirp Signal-White Noise}

As another interesting example, consider the analysis of a pure chirp signal $\left(\omega_{0}>0\right)$

$$
s(t)=a_{0} \cos \left(\omega_{0} t^{2}\right)
$$

over the time window $t \in\left[1 /\left(2^{M} \omega_{0}\right), 1 /\left(2 \omega_{0}\right)\right]$. Over this span the instantaneous frequency

$$
\omega_{i}(t)=2 \omega_{0} t
$$

of (86) will, for each $t$, fall within the frequency interval $\left[2^{l-M}, 1\right]$ (again taking $\Omega_{c}=1$ and avoiding the baseband for simplicity of exposition). In order to model the way in which the ESA computation passes between channels, it is convenient to first consider the signal to be noisefree. Then, the performance of the multiband ESA can be analyzed in the signal-plus-noise case using this information.

This time we utilize the Gabor wavelet configuration. From (36) and (37), the normalized energies of the response of the filter $(80)$ to the chirp (86) at time $t$ are

$$
\begin{aligned}
& \frac{\Psi\left(s_{m}\right)}{\left|G_{m}\left(\omega_{m}\right)\right|^{2}} \approx a_{0}^{2}\left(2 \omega_{0} t\right)^{2} \cdot\left|\frac{G_{m}\left(2 \omega_{0} t\right)}{G_{m}\left(\omega_{m}\right)}\right|^{2} \\
& \frac{\Psi\left(\dot{\mathrm{s}}_{m}\right)}{\left|G_{m}\left(\omega_{m}\right)\right|^{2}} \approx a_{0}^{2}\left(2 \omega_{0} t\right)^{4} \cdot\left|\frac{G_{m}\left(2 \omega_{0} t\right)}{G_{m}\left(\omega_{m}\right)}\right|^{2} .
\end{aligned}
$$

Selecting the (index of the) analysis channel as a function of time then follows from (72) and (87):

$$
m^{*}(t)=\underset{1 \leq m \leq M}{\arg \max }\left|\frac{G_{m}\left(2 \omega_{0} t\right)}{G_{m}\left(\omega_{m}\right)}\right|^{2}=\underset{1 \leq m \leq M}{\arg \min }\left|2 \omega_{0} t-\omega_{m}\right|
$$

if it is assumed that

$$
\left|\frac{G_{m}\left(2 \omega_{0} t\right)}{G_{m}\left(\omega_{m}\right)}\right|^{2} \approx \exp \left\{-2\left(\frac{2 \omega_{0} t-\omega_{m}}{\sigma_{m}}\right)^{2}\right\}
$$

From (75) and (81), it follows that $m=m *(t)$ for

$$
\left(\frac{9}{16 \omega_{0}}\right) 2^{-m}<t \leq\left(\frac{9}{8 \omega_{0}}\right) 2^{-m}
$$

not surprisingly, higher-frequency filters analyze the signal over larger time windows, since they are allocated larger pieces of the spectrum. Fig. 8 depicts the intervals over which each filter $g_{m}(t)$ will be "active," viz., for which $m=m^{*}(t)$ in (88), for $m=1,2,3,4=M-1$ using the set of 5 wavelets depicted in Fig. 7, after appropriately scaling the filters to cover the frequency range $[0,1]$. Note again that the example was designed such that the baseband filter does not become active.

The effects of noise on the multiband energies of the chirp signal are considered next. This time, assume the signal $s(t)$ to be immersed in zero-mean, additive Gaussian white noise with power spectrum

$$
\Phi(\omega)=\frac{\eta}{\sqrt{2 \pi}} ; \quad \omega \in \boldsymbol{R}
$$

Of course, for this noise model, image prefiltering using bandpass filters is an absolute necessity, since the energy operator $\Psi$ cannot be meaningfully applied to a white noise signal.

In-Band Energy Moments: Assuming that $m=m^{*}(t)$, i.e., that at time $t$ the channel filter $G_{m}(\omega)$ contains the instantaneous frequency $\omega_{i}(t)=2 \omega_{0} t$ (regardless of whether $g_{m}(t)$ is correctly selected as the analyzing wavelet), then from (43), (55)-(58), the normalized energy moments are

$$
\begin{aligned}
\frac{\mathrm{E}\left[\Psi\left(f_{m}\right)\right]}{\left|G_{m}\left(\omega_{m}\right)\right|^{2}} \approx & \left(2 \omega_{0} t\right)^{2}\left(a_{0}^{2}+4 \eta \sigma_{m}\right) \\
& \cdot \exp \left\{-2\left(\frac{2 \omega_{0} t-\omega_{m}}{\sigma_{m}}\right)^{2}\right\} \\
\frac{E\left[\Psi\left(\dot{f}_{m}\right)\right]}{\left|G_{m}\left(\omega_{m}\right)\right|^{2}} \approx & \left(2 \omega_{0} t\right)^{4}\left(a_{0}^{2}+4 \eta \sigma_{m}\right) \\
& \cdot \exp \left\{-2\left(\frac{2 \omega_{0} t-\omega_{m}}{\sigma_{m}}\right)^{2}\right\}
\end{aligned}
$$

and

$$
\begin{aligned}
\frac{\operatorname{Var}\left[\Psi\left(f_{m}\right)\right]}{\left|G_{m}\left(\omega_{m}\right)\right|^{4}} \approx & 2 \eta \sigma_{m}\left(2 \omega_{0} t\right)^{4}\left(a_{0}^{2}+2 \eta \sigma_{m}\right) \\
& \cdot \exp \left\{-4\left(\frac{2 \omega_{0} t-\omega_{m}}{\sigma_{m}}\right)^{2}\right\}
\end{aligned}
$$




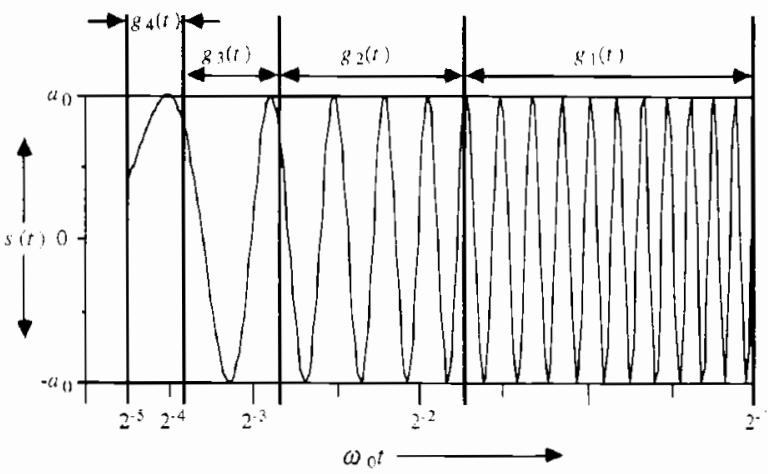

Fig. 8. Division of chirp signal $s(t)$ given by $(86)$ into time intervals over which different Gabor wavelets from the set of five depicted in Fig. 6 will be "active."

$$
\begin{aligned}
\frac{\operatorname{Var}\left[\Psi\left(f_{m}\right)\right]}{\mid G_{m}\left(\omega_{m}\right)^{4}} \approx & 2 \eta \sigma_{m}\left(2 \omega_{0} t\right)^{8}\left(a_{0}^{2}+2 \eta \sigma_{m}\right) \\
& \cdot \exp \left\{-4\left(\frac{2 \omega_{0} t-\omega_{m}}{\sigma_{m}}\right)^{2}\right\}
\end{aligned}
$$

Out-of-Band Energy Moments: Suppose $q \neq m^{*}(t)$ at time $t$ (although it is possible that $g_{q}(t)$ may be selected as the analyzing wavelet). To determine the normalized out-ofband energy moments (energy moments from band $q$ ), use the approximation (41) with $\alpha=\omega_{q}$

$$
\begin{aligned}
& \frac{\mathrm{E}\left[\Psi\left(n_{q}\right)\right]}{\left|G_{q}\left(\omega_{q}\right)\right|^{2}} \approx 2 \eta \sigma_{q} \omega_{q}^{2} \\
& \frac{\mathrm{E}\left[\Psi\left(\dot{n}_{q}\right)\right]}{\left|G_{q}\left(\omega_{q}\right)\right|^{2}} \approx 2 \eta \sigma_{q} \omega_{q}^{4}
\end{aligned}
$$

and

$$
\begin{aligned}
& \frac{\operatorname{Var}\left[\Psi\left(n_{q}\right)\right]}{\left|G_{q}\left(\omega_{q}\right)\right|^{4}} \approx 4 \eta^{2} \sigma_{q}^{2} \omega_{q}^{4} \\
& \frac{\operatorname{Var}\left[\Psi\left(\dot{n}_{q}\right)\right]}{\left|G_{q}\left(\omega_{q}\right)\right|^{4}} \approx 4 \eta^{2} \sigma_{q}^{2} \omega_{q}^{8} .
\end{aligned}
$$

For narrowband channel filters, these approximations yield accurate approximations for the moments of the responses of any channel to a pure noise stimulus.

Channel Selection: Observing from (88) that $2 \omega_{0} t>$ $(9 / 8) 2^{-m}$ for $m=m^{*}(t)$, the ratios of (89) to (93) and (90) to (94) are respectively bounded below by

$$
\begin{aligned}
& \left(\frac{9}{16}\right) 2^{3 q-2 m} \sqrt{\ln 2} s n r_{0} \\
& \left(\frac{9}{16}\right)^{2} 2^{5 q-4 m} \sqrt{\ln 2} s n r_{0}
\end{aligned}
$$

where

$$
s n r_{0}=\frac{a_{0}^{2}}{\eta} .
$$

For $s n r_{0}$ large, the in-band channel will dominate the other (noise) channels. Thus, the channel containing the local signal frequencies (the in-band channel) will be selected as the analysis channel with a high probability. However, from (97) and (98) it may again be observed that the energy operator has increased sensitivity when the signal to be detected is lower frequency, and immersed in highfrequency noise [see the discussion below (72)].

ESA Computation: If the correct channel is selected, then since the ratio of the square of (89) to (91), and of the square of (90) to (92) are both bounded below by

$$
2^{m+2} \sqrt{\ln 2} s n r_{0}
$$

then for reasonably high $s n r_{0}$ the normalized energies satisfy

$$
\begin{aligned}
& \frac{\Psi\left(s_{m}\right)}{\left|G_{m}\left(\omega_{m}\right)\right|^{2}} \approx a_{0}^{2}\left(2 \omega_{0} t\right)^{2} \\
& \frac{\Psi\left(\dot{s}_{m}\right)}{\left|G_{m}\left(\omega_{m}\right)\right|^{2}} \approx a_{0}^{2}\left(2 \omega_{0} t\right)^{4} .
\end{aligned}
$$

Hence, when $s n r_{0}$ is large, the computation of the ESA using the channel that yields the maximum normalized energy response will yield the desired ESA computations

$$
\begin{aligned}
& \hat{\omega}_{i}^{2}(t) \approx\left(2 \omega_{0} t\right)^{2} \\
& \hat{a}^{2}(t) \approx a_{0}^{2}
\end{aligned}
$$

for each $\left.t \in\left[1 / 2^{M} \omega_{0}\right), 1 /\left(2 \omega_{0}\right)\right]$.

\section{Simulation Results}

In order to verify the predicted results developed in the preceding, the multiband ESA was implemented and applied to several interesting signals, using the MATLAB Signal Processing Toolkit [25]. In all of the examples the filters in the Littlewood-Paley filter bank (80) were approximated by equiripple (Chebyshev) FIR bandpass filters numerically designed using the Parks-McClellan algorithm [26]. The filters are all of order 101. As in the preceding analysis, five channel filters were used (including baseband). The frequency response magnitudes of the five filters are shown in Fig. 9; note that unity-magnitude passbands were used to simplify the computation of (72). The ESA results in the simulations were computed using the discrete-time Teager-Kaiser energy operator [9]; while the theoretical development of the discrete-time operator/ ESA is nontrivial (and hence not developed here), implementation of it requires only minor modifications relating primarily to sampling approximations that do not present an issue in the context of these simulations.

In the first simulation example, a digital implementation of the multiband ESA was applied to a signal composed of a sum of two sinusoidal functions, one with magnitude 1.0 and $1000 \mathrm{~Hz}$ frequency, and the other with magnitude 0.2 and a $2000 \mathrm{~Hz}$ frequency. The signal (and all other simulated signals) were sampled at a rate of $10,000 \mathrm{~Hz}$. This example corresponds to the analysis in Section VI-A, where one of the sinusoid components may be considered to be noise. Fig. 10(a) and (b) show the computed ESA results using the multiband ESA. Both the 


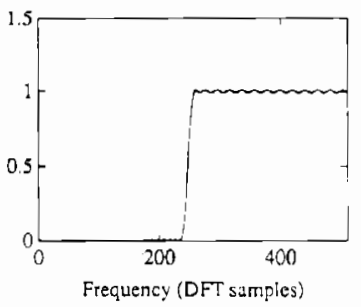

(a)

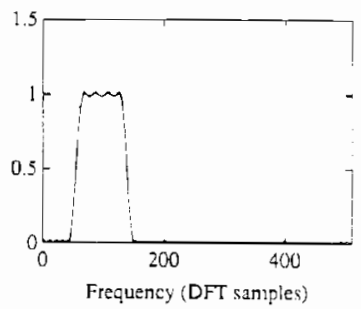

(c)

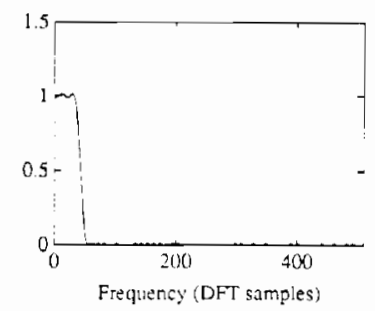

(c)

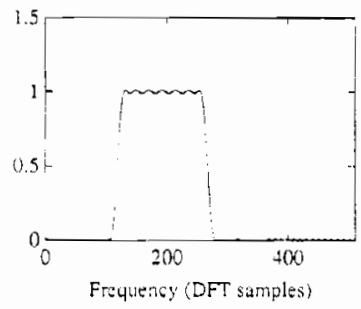

(b)

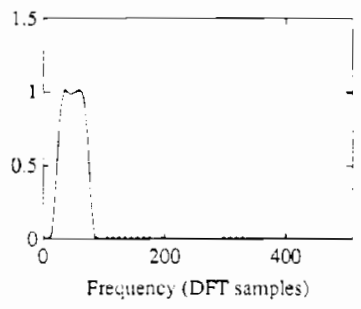

(d)

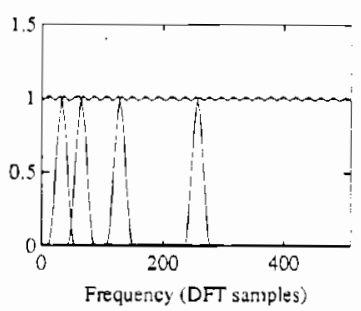

(f)
Fig. 9. (a)-(e) Magnitude responses of the filters composing the multiband implementation used in the simulations. The filters were designed using the Parks-McClellan FIR equiripple (Chebyshev) algorithm. (f) Plot of ail five magnitude responses showing the small amount of band overlap.

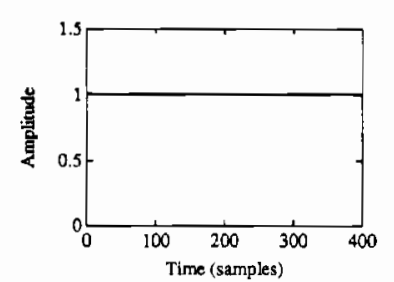

(a)

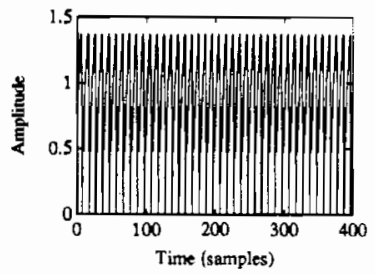

(c)

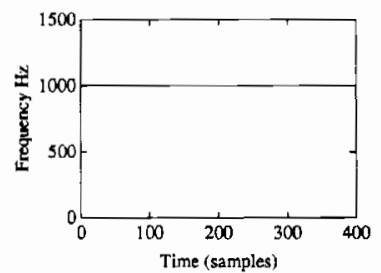

(b)

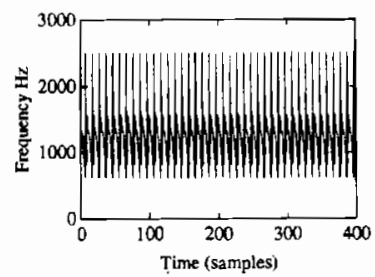

(d)
Fig. 10. ESA applied to sum of two sinusoidal components with (magnitude, frequency) $=(1.0,1000 \mathrm{~Hz})$ and $(0.2,2000 \mathrm{~Hz})$. (a), (b) Amplitude and frequencies computed using the multiband ESA. (c). (d) Amplitude and frequencies computed using the ESA without filtering.

signal amplitude and the signal frequency of the component having the largest energy were approximated with a high accuracy, although a small ripple in the computed result may be observed. By comparison, Fig. 10(c) and (d) show the computed ESA results without filtering. In this case, the ESA result is extremely poor and cannot even be considered to be useful. Fig. 11(a)-(d) depicts a similar result, only this time the signal is a unity-magnitude $1000 \mathrm{~Hz}$ sinusoid added to a $1200 \mathrm{~Hz}$ sinusoid with magnitude 0.3 . Similar high-quality results were obtained, demonstrating the capability of the multiband ESA to provide excellent resolution, provided that the signal components do not fall within the same band.

Fig. 12(a) depicts a chirp signal having a 3000 $\mathrm{Hz}$ /second sweep rate ( $1000 \mathrm{~Hz}$ initial frequency), immersed in additive Gaussian white noise. The signal-tonoise ratio (SNR) is $15 \mathrm{~dB}$. Fig. 12(b) and (c) depict the result of the multiband ESA computation; again, the results are highly accurate, although there is noticeable fluctuation in the estimate of the amplitude. If plotted, the estimated instantaneous frequencies could not be discerned, since they fall directly on the plot of the computed result. The root-mean-squared (rms) error relative to the ideal was computed for both the AM and FM estimates, and found to be 0.039 ( $\mathrm{AM}$ rms error) and $11.4 \mathrm{~Hz}$ (FM rms error). By comparison, Fig. 12(d) and (e) shows the ESA computation obtained without any filtering. The computed rms values were 0.77 (AM rms error) and $594.17 \mathrm{~Hz}$ (FM rms error). Clearly, the multiband approach affords a vast improvement. Both results (with and without filtering) could be improved by some kind of postprocessing, e.g., low-pass or median post-smoothing, but, in any case far superior results are clearly obtained by using the multiband approach to isolate the signal information.

Fig. 13(a) depicts another chirp signal, with initial frequency $2000 \mathrm{~Hz}$ and a $3000 \mathrm{~Hz} / \mathrm{sec}$ sweep, only this time with a $20 \mathrm{~Hz}$ amplitude modulation. The signal was immersed in noise as depicted in Fig. 13(b) $(S N R=15 \mathrm{~dB})$. The ESA results with filtering are shown in Fig. 13(c) and (d) $(\mathrm{AM}$ rms error $=0.046$, FM rms error $=32.4 \mathrm{~Hz}$ ) and without filtering in Fig. 13(e) and (f) (AM rms error $=0.27$, FM rms error $=543.41 \mathrm{~Hz}$ ). Again, the multiband ESA results present enormous improvement over the simple ESA computation.

Finally, Fig. 14(a) depicts an AM $(20 \mathrm{~Hz})$ noisy chirp signal $(\mathrm{SNR}=15 \mathrm{~dB})$ with initial frequency $2400 \mathrm{~Hz}$ and a $3000 \mathrm{~Hz} / \mathrm{sec}$ sweep rate. Fig. 14(b) and (c) depict the results of the multiband ESA computation. The computed errors were found to be 0.044 (AM rms error) and 59.8 $\mathrm{Hz}$ (FM rms error). This last example is presented since the instantaneous frequencies of the chirp signal component sweep well across a transition from one channel passband to another, demonstrating the sustained, continuous performance of the multiband ESA as predicted in Section V-C. Fig. 14(d) depicts the transitions between the two highest frequency channels (labeled as channels 2 and 1) that were automatically selected by the multiband ESA algorithm according to the maximum criterion in (72). Clearly, (72) oscillates quite a bit between channels 2 and 1 near the theoretical cross-over point of $2500 \mathrm{~Hz}$. 


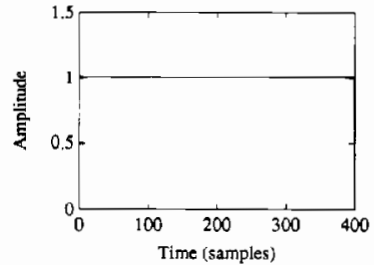

(a)

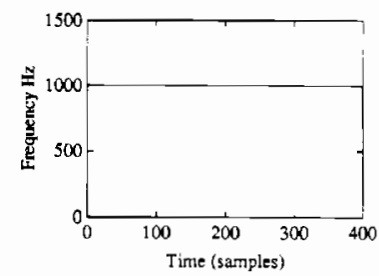

(b)

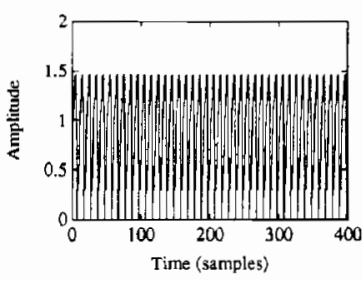

(c)

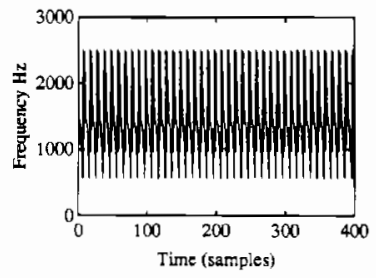

(d)

Fig. 11. ESA applied to sum of two sinusoidal components with (magnitude, frequency) $=(1.0,1000 \mathrm{~Hz})$ and $(0.3,1200 \mathrm{~Hz}$ ). (a), (b) Amplitude and frequencies computed using the multiband ESA. (c), (d) Amplitude and frequencies computed using the ESA without filtering.

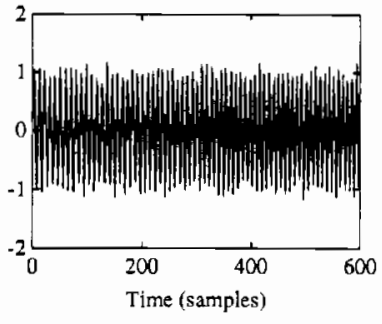

(a)

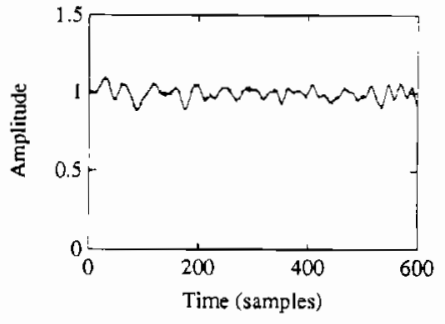

(b)

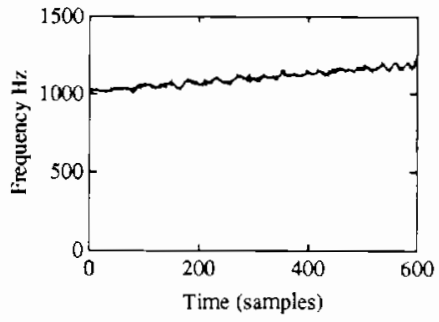

(c)

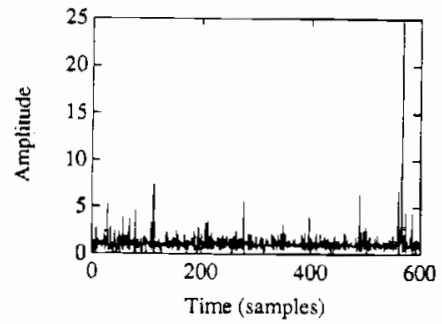

(d)

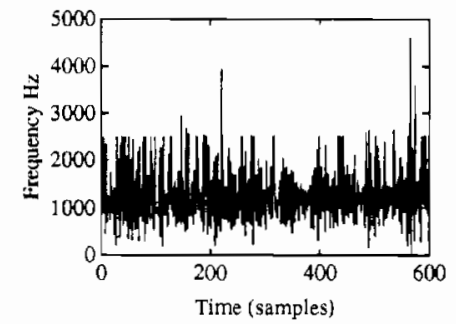

(e)

Fig. 12. ESA computation applied to the noisy chirp signal depicted in (a). (b), (c) Amplitude and frequencies computed using the multiband ESA. (d), (e) Amplitude and frequencies computed using the ESA without filtering.

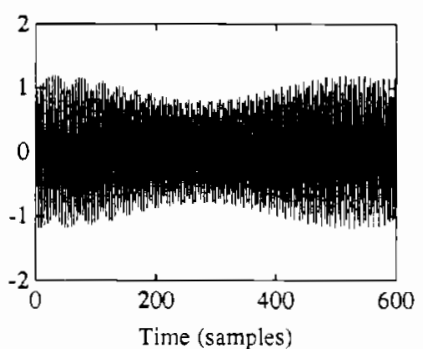

(a)

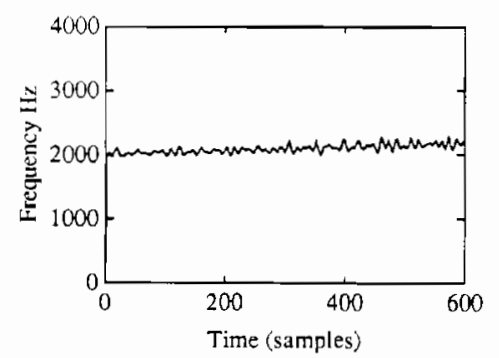

(d)

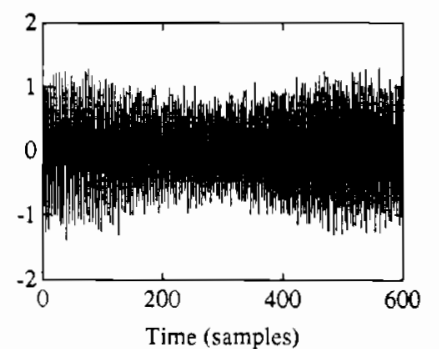

(b)

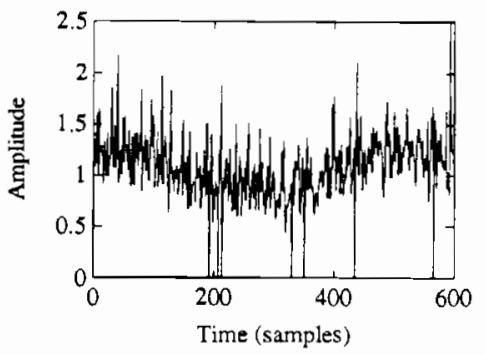

(e)

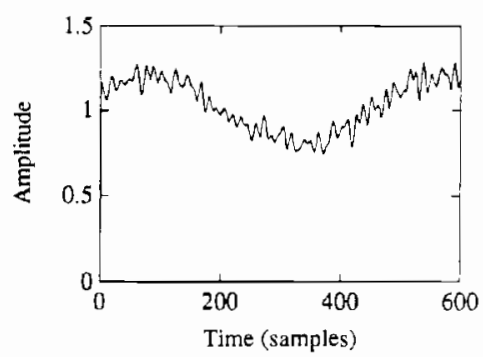

(c)

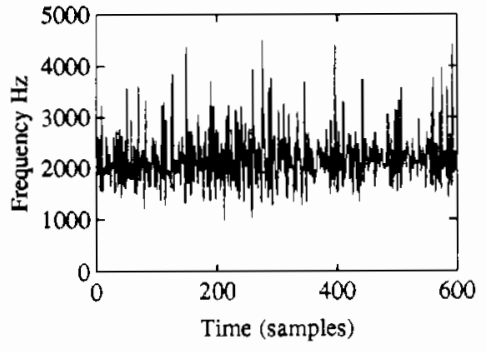

(f)

Fig. 13. ESA computation applied to AM chirp signal depicted in (a) immersed in noise as in (b). (c). (d) Amplitude and frequencies computed using the multiband ESA. (e), (f) Amplitude and frequencies computed using the ESA without filtering. 


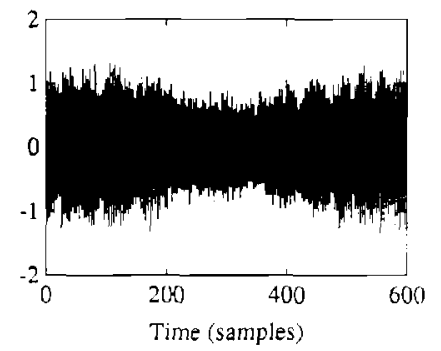

(a)

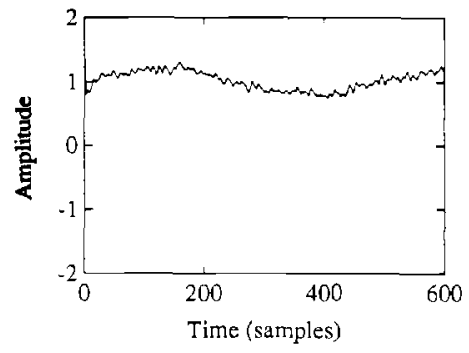

(b)

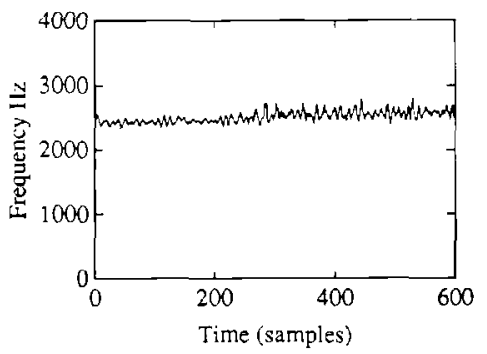

(c)

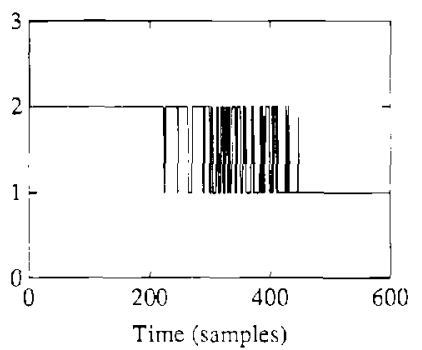

(d)

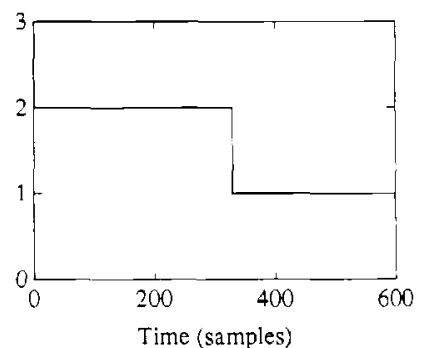

(e)

Fig. 14. ESA computation applied to noisy AM chirp signal depicted in (a), with instantaneous frequency that sweeps across filter passbands. (b), (c) Amplitude and frequencies computed using the multiband ESA. (d), (e) transitions between channels 2 and 1 according to (72).

This is not surprising, since the filter transition width is about $50 \mathrm{~Hz}$, and the chirp sweep is fairly slow. Of course, the oscillation between channels is not undesirable; rather, it is a natural consequence of the maximizing criterion (72), and demonstrates the robustness of the multiband ESA for the main goal AM/FM demodulation in the presence of noise. Finally, Fig. 14(e) shows that a single switch from channel 2 to channel 1 does occur (at 2500 $\mathrm{Hz}$ ) when the multiband ESA is applied to the same signal 14(a), but without noise.

\section{Conclusion}

This paper has developed a multiband/wavelet-like approach for capturing AM-FM information from noisy modulated signals. An in-depth statistical analysis was presented of the nonlinear energy operator $\Psi(s)=(\dot{s})^{2}-$ $s \ddot{s}$, and of a related energy separation algorithm (ESA). It was demonstrated that overall approach is greatly improved by first filtering the observed signal with multiple bandpass filters, and at each instant analyzed using the dominant local channel response. Importantly, uniform worst-case performance across the spectrum can only be attained by using a constant- $Q$, or multiscale wavelet-like filter bank.

Future work that remains to be accomplished includes the analysis of digital implementations of the systems described herein, using the discrete-time Teager-Kaiser energy operator [5], and also extensions of the paradigm that will allow for the analysis of multicomponent AM-FM signals of the form

$$
s(t)=\sum_{k=1}^{K} a_{k}(t) \cos \left[\phi_{k}(t)\right]
$$

The problem then involves the tracking of multiple components that may merge, vanish, or possibly contains discontinuities. Although the problem is difficult, we suspect that the applications of the model (99) will be very widespread.

\section{APPENDIX A}

Proof of Theorem 1: We first note that by Taylor's theorem with remainder

$$
\phi(t-x)=\phi(t)-x \dot{\phi}(t)+Q_{\phi}(t, x)
$$

where

$$
Q_{\phi}(t, x)=x^{2} \int_{0}^{1}(1-b) \ddot{\phi}(t-b x) d b
$$

From (1) and (A.1) we have

$$
\begin{aligned}
s_{\sigma}(t)= & a(t) \cos [\phi(t)] * g_{\sigma}(t) \\
= & \left(\frac{1}{2}\right) \int_{R} g_{\sigma}(x) a(t-x) \exp [j \phi(t)] \\
& \cdot \exp [-j x \dot{\phi}(t)] \exp \left[j Q_{\phi}(t, x)\right] d x \\
& +\left(\frac{1}{2}\right) \int_{R} g_{\sigma}(x) a(t-x) \exp [-j \phi(t)] \\
& \cdot \exp [j x \dot{\phi}(t)] \exp \left[-j Q_{\phi}(t, x)\right] d x
\end{aligned}
$$


and from (31) we have

$$
\begin{aligned}
\hat{s}_{\sigma}(t)= & a(t)\left|G_{\sigma}\left[\omega_{i}(t)\right]\right| \cos \left\{\phi(t)+\angle G_{\sigma}\left[\omega_{i}(t)\right]\right\} \\
= & \left(\frac{1}{2}\right) \int_{R} g_{\sigma}(x) a(t) \exp [j \phi(t)] \exp [-j x \dot{\phi}(t)] d x \\
& +\left(\frac{1}{2}\right) \int_{R} g_{\sigma}(x) a(t) \exp [-j \phi(t)] \exp [j x \dot{\phi}(t)] d x .
\end{aligned}
$$

By the triangle inequality the difference between (A.3) and (A.4) is bounded as

$$
\begin{aligned}
\mathcal{E}_{s}(t)= & \left|s_{\sigma}(t)-\hat{s}_{\sigma}(t)\right| \\
\leq & \left(\frac{1}{2}\right) \int_{R}\left|g_{\sigma}(x)\right| \mid a(t-x) \exp \left[j Q_{\phi}(t, x)\right] \\
& -a(t) \mid d x \\
& +\left(\frac{1}{2}\right) \int_{R}\left|g_{\sigma}(x)\right| \mid a(t-x) \exp \left[-j Q_{\phi}(t, x)\right] \\
& -a(t) \mid d x .
\end{aligned}
$$

Both terms in (A.5) may be further bounded identically

$$
\begin{aligned}
\int_{R}\left|g_{\sigma}(x)\right|\left|a(t-x) \exp \left[ \pm j Q_{\phi}(t, x)\right]-a(t)\right| d x \\
\leq \quad \int_{R}\left|g_{\sigma}(x)\right||a(t-x)|\left|\exp \left[ \pm j Q_{\phi}(t, x)\right]-1\right| d x \\
\quad+\int_{R}\left|g_{\sigma}(x)\right||a(t-x)-a(t)| d x \\
\leq a_{\max } \int_{R}\left|g_{\sigma}(x)\right|\left|Q_{\phi}(t, x)\right| d x+\int_{R}\left|g_{\sigma}(x)\right| \\
\quad \cdot|a(t-x)-a(t)| d x \\
=\mathcal{E}_{s, \phi}(t)+\mathcal{E}_{s, a}(t) .
\end{aligned}
$$

However, by again applying Taylor's theorem with remainder

$$
\begin{aligned}
|a(t-x)-a(t)|= & \left|-x \int_{0}^{1} \dot{a}(t-b x) d b\right| \\
& \leq|x| \int_{0}^{1}|\dot{a}(t-b x)| d b
\end{aligned}
$$

we obtain by an interchange of integrals and the Cauchy-Schwarz inequality.

Furthermore, from (A.2), (A.6), and (2) we have

$$
\begin{aligned}
\mathcal{E}_{5, \phi}(t) \leq & a_{\max } \int_{0}^{1}(1-b) \int_{R} x^{2}\left|g_{o}(x)\right||\ddot{\phi}(t-b x)| d x d b \\
\leq & a_{\max } \int_{0}^{1}(1-b)\left[\int_{R} x^{4}\left|g_{\sigma}(x)\right|^{2} d x\right]^{1 / 2} \\
& \cdot\left[\int_{R}|\ddot{\phi}(t-b x)|^{2} d t\right]^{1 / 2} d b \\
= & a_{\max } \Delta_{2}\left(g_{\sigma}\right) \delta\left(\omega_{i}\right) \int_{0}^{1}(1-b) b^{-1 / 2} d b \\
= & \frac{4}{3} a_{\max } \Delta_{2}\left(g_{\sigma}\right) \delta\left(\omega_{i}\right) .
\end{aligned}
$$

The proof is completed by combining (A.5), (A.6), and (A.8)

\section{APPENDIX B}

The proof of Theorem 2 requires the following Lemma:

Lemma 1: Suppose that the AM-FM signal $s(t)=a(t)$ $\cos [\phi(t)]$ is applied to two linear systems $g_{1}: \boldsymbol{R} \rightarrow \boldsymbol{R}$ and $g_{2}: \boldsymbol{R} \rightarrow \boldsymbol{R}$. Let

$k(t)=\left[g_{1}(t) * s(t)\right]\left[g_{2}(t) * s(t)\right]$

and

$$
\begin{aligned}
\hat{k}(t)= & a^{2}(t)\left|G_{1}[\dot{\phi}(t)]\right|\left|G_{2}[\dot{\phi}(t)]\right| \\
& \cdot \cos \left\{\phi(t)+\angle G_{1}[\dot{\phi}(t)]\right\} \\
& \cdot \cos \left\{\phi(t)+\angle G_{2}[\dot{\phi}(t)]\right\} .
\end{aligned}
$$

Then,

$$
\begin{aligned}
\varepsilon_{k}(t)= & |k(t)-\hat{k}(t)| \leq \frac{4}{3}\left(a_{\max }\right)^{2} \cdot\left[\bar{g}_{1} \Delta_{2}\left(g_{2}\right)\right. \\
& \left.+\bar{g}_{2} \Delta_{2}\left(g_{1}\right)\right] \cdot \delta\left(\omega_{i}\right) \\
& +2 a_{\max } \cdot\left[\bar{g}_{1} \Delta_{1}\left(g_{2}\right)+\bar{g}_{2} \Delta_{1}\left(g_{1}\right)\right] \cdot \delta(\alpha)
\end{aligned}
$$

where $\Delta_{m}(\cdot), \delta(\cdot)$ and $\bar{g}$ are given in (33) and (38).

Proof: Write

$$
k(t)=\left(\frac{1}{4}\right) \sum_{m, n \in\{-1,1\}}\left\{g_{1}(t) * a(t) \exp [j m \phi(t)]\right\}
$$$$
\cdot\left\{g_{2}(t) * a(t) \exp [j n \phi(t)]\right\}
$$

$$
\begin{aligned}
\mathcal{E}_{s, a}(t) & \leq \int_{0}^{1} \int_{R}|x|\left|g_{\sigma}(x)\right||\dot{a}(t-b x)| d x d b \\
& \leq \int_{0}^{1}\left[\int_{R} x^{2}\left|g_{\sigma}(x)\right|^{2} d x\right]^{1 / 2}\left[\int_{R}|\dot{a}(t-b x)|^{2} d x\right]^{1 / 2} d b \\
& =\Delta_{1}\left(g_{\sigma}\right) \delta(a) \int_{0}^{1} s^{-1 / 2} d s=2 \Delta_{1}\left(g_{\sigma}\right) \delta(a) .
\end{aligned}
$$


and also

$$
\begin{aligned}
\hat{k}(t)= & \left(\frac{1}{4}\right) \sum_{m, n \in\{-1.1\}} a^{2}(t) G_{1}[m \dot{\phi}(t)] G_{2}[n \dot{\phi}(t)] \\
& \cdot \exp [j(m+n) \phi(t)]
\end{aligned}
$$

so the following bound on the difference holds:

$$
\mathcal{E}_{k}(t) \leq\left(\frac{1}{4}\right) \sum_{m, n \in\{-1,1\}} \mathcal{E}_{k}(t ; m, n)
$$

where

$$
\begin{aligned}
\mathcal{E}_{k}(t ; m, n)= & \mid\left\{g_{1}(t) * a(t) \exp [j m \phi(t)]\right\}\left\{g_{2}(t) * a(t)\right. \\
& \cdot \exp [j n \phi(t)]\}-a^{2}(t) G_{1}[m \dot{\phi}(t)] \\
& \cdot G_{2}[n \dot{\phi}(t)] \exp [j(m+n) \phi(t)] \mid .
\end{aligned}
$$

Now,

$$
\begin{aligned}
\mathcal{E}_{k}(t ; m, n)= & \mid \iint_{R^{2}} g_{1}(x) g_{2}(y) a(t-x) a(t-y) \\
& \cdot \exp \{j(m+n) \phi(t)-j(m x+n y) \dot{\phi}(t) \\
& \left.+j\left[m Q_{\phi}(t, x)+n Q_{\phi}(t, y)\right]\right\} d x d y \\
& -\iint_{R^{2}} g_{1}(x) g_{2}(y) a^{2}(t) \cdot \exp \{j(m+n) \\
& \cdot \phi(t)-j(m x+n y) \dot{\phi}(t)\} d x d y \mid \\
\leq & \iint_{R^{2}}\left|g_{1}(x)\right|\left|g_{2}(y)\right| \mid a(t-x) a(t-y) \\
& \cdot \exp \left\{j\left[m Q_{\sigma}(t, x)+n Q_{\phi}(t, y)\right]\right\} \\
& -a^{2}(t) \mid d x d y \\
\leq & \mathcal{E}_{k, \phi}(t ; m, n)+\mathcal{E}_{k, a}(t ; m, n),
\end{aligned}
$$

where

$$
\begin{aligned}
\mathcal{E}_{k, \phi}(t ; m, n) & \\
= & \iint_{R^{2}}\left|g_{1}(x)\right|\left|g_{2}(y)\right||a(t-x) a(t-y)| \\
& \cdot\left|\exp \left\{j\left[m Q_{\phi}(t, x)+n Q_{\phi}(t, y)\right]\right\}-1\right| d x d y \\
\leq & \left(a_{\max }\right)^{2} \iint_{R^{2}}\left|g_{1}(x)\right|\left|g_{2}(y)\right|\left|Q_{\phi}(t, x)\right| d x d y \\
& +\left(a_{\max }\right)^{2} \iint_{R^{2}}\left|g_{1}(x)\right|\left|g_{2}(y)\right|\left|Q_{\phi}(t, y)\right| d x d y \\
= & \left(a_{\max }\right)^{2}\left[\bar{g}_{1} \int_{R}\left|g_{2}(y)\right|\left|Q_{\phi}(t, y)\right| d y+\bar{g}_{2}\right. \\
& \left.\cdot \int_{R}\left|g_{1}(x)\right|\left|Q_{\phi}(t, x)\right| d x\right]
\end{aligned}
$$

$\mathcal{E}_{k, a}(t ; m, n)$

$$
=\iint_{R^{2}}\left|g_{1}(x)\right|\left|g_{2}(y)\right| \mid a(t-x) a(t-y)
$$$$
-a^{2}(t) \mid d x d y
$$$$
\leq \iint_{R^{2}}\left|g_{1}(x)\right|\left|g_{2}(y)\right||a(t-x)| \mid a(t-y)
$$$$
\text { - } a(t) \mid d x d y
$$$$
+\iint_{R^{2}}\left|g_{1}(x)\right|\left|g_{2}(y)\right||a(t)| \mid a(t-x)
$$$$
-a(t) \mid d x d y
$$

$$
\begin{aligned}
\leq & a_{\max }\left[\bar{g}_{1} \int_{R}\left|g_{2}(y)\right||a(t-y)-a(t)| d y\right. \\
& \left.+\bar{g}_{2} \int_{R}\left|g_{1}(x)\right||a(t-x)-a(t)| d x\right] .
\end{aligned}
$$

However, from (A.6) and (A.8) we have that

$$
\int_{R}\left|g_{2}(y)\right|\left|Q_{\phi}(t, y)\right| d y \leq \frac{4}{3} \Delta_{2}\left(g_{2}\right) \delta\left(\omega_{i}\right)
$$

so that (B.2) becomes

$$
\varepsilon_{k, \phi}(t ; m, n) \leq \frac{4}{3}\left(a_{\max }\right)^{2}\left[\bar{g}_{1} \Delta_{2}\left(g_{2}\right)+\bar{g}_{2} \Delta_{2}\left(g_{1}\right)\right] \delta\left(\omega_{i}\right),
$$

We also have from (A.6) and (A.7) that

$$
\int_{R}\left|g_{2}(y)\right||a(t-y)-a(t)| d y \leq 2 \Delta_{1}\left(g_{2}\right) \delta(a) .
$$

so that (B.3) becomes

$$
\varepsilon_{k, 0}(t ; m, n) \leq 2 a_{\max }\left[\bar{g}_{1} \Delta_{1}\left(g_{2}\right)+\bar{g}_{2} \Delta_{1}\left(g_{1}\right)\right] \delta(a) .
$$

Combining (B.1), (B.4), and (B.5) completes the proof.

Proof of Theorem 2: From (3) and (A.1) we have

$$
\Psi\left(s_{\sigma}\right)=\left(\dot{s}_{\sigma}\right)^{2}-s_{\sigma} \ddot{s}_{\sigma}=(s * \dot{g})^{2}-(s * g)(s * \ddot{g}) \text {. }
$$

The proof follows by applying Lemma 1 to the first term of (B.6) with $g_{1}=g_{2}=\dot{g}_{\sigma}$, by also applying Lemma 1 to the second term of (B.6) with $g_{1}=g_{\sigma}, g_{2}=\ddot{g}$, and by taking the differences of the respective approximations to yield (35). The bound (39) follows easily by applying the triangle inequality to the sum of the errors of these approximations.

Note: Equation (B.6) has also been used in [27] as an alternative way of applying the energy operator to sampled, bandpass-filtered speech signals, as a means for reducing the discretization effects introduced by using discrete derivative operators to approximate $\Psi$. 


\section{Appendix C}

Proof of Theorem 3: Clearly

$$
\begin{aligned}
& \varepsilon_{R_{\sigma}}^{(k)}(\alpha) \leq\left.\frac{1}{2 \pi} \int_{R}\left|\omega^{2 k}-\alpha^{2 k}\right| \frac{G_{\sigma}(\alpha)}{G_{\sigma}\left(\omega_{0}\right)}\right|^{2}\left|G_{\sigma}(\omega)\right|^{2} \Phi(\omega) \\
& \cdot d \omega \leq A+B,
\end{aligned}
$$

where

$$
A=\frac{1}{2 \pi} \int_{R}\left|\omega^{2 k}-2^{2 k}\right|\left|G_{\sigma}(\omega)\right|^{2} \Phi(\omega) d \omega
$$

and

$$
B=\left.\left.\frac{1}{2 \pi} \alpha^{2 k}|1-| \frac{G_{a}(\alpha)}{G_{\sigma}\left(\omega_{c}\right)}\right|^{2}\left|\cdot \int_{R}\right| G_{\sigma}(\omega)\right|^{2} \Phi(\omega) d \omega .
$$

Now $A=0$ when $k=0$, hence assume $k \geq 1$. Using the identities,

$$
\begin{aligned}
\omega^{2 k}-\alpha^{2 k}= & (\omega-\alpha) \sum_{i=0}^{2 k-1} \omega^{2 k-1-i} \alpha^{i}=(\omega+\alpha) \\
& \cdot \sum_{i=0}^{2 k-1} \omega^{2 k-1-i}(-\alpha)^{i}
\end{aligned}
$$

and from (29) it follows that

$$
\begin{aligned}
A \leq & \frac{1}{2 \pi} \Phi_{\max } \int_{R}\left[|\omega-\alpha|\left|H_{\sigma}\left(\omega-\omega_{c}\right)\right|^{2}\right. \\
& \left.+|\omega+\alpha|\left|H_{\sigma}\left(\omega+\omega_{c}\right)\right|^{2}\right] \\
& \cdot \sum_{i=0}^{2 k-1}\left|\omega^{2 k-1-i} \alpha^{i}\right| d \omega \\
= & \frac{1}{\pi} \Phi_{\max } \int_{R}|\omega-\alpha|\left|H_{\sigma}\left(\omega-\omega_{c}\right)\right|^{2} \\
& \cdot \sum_{i=0}^{2 k-1}\left|\omega^{2 k-1-i} \alpha^{i}\right| d \omega
\end{aligned}
$$

where the equality follows from the symmetry of $H(\omega)$. Simple algebraic inequalities yield

$$
\sum_{i=0}^{2 k-1}\left|\omega^{2 k-1-i} \alpha^{i}\right| \leq 2 k\left(|\omega|^{2 k-1}+|\alpha|^{2 k-1}\right)
$$

Substituting (C.2) into (C.1) and applying the CauchySchwarz inequality yields

$$
\begin{aligned}
A \leq & 4 k \Phi_{\max }\left[\frac{1}{2 \pi} \int_{R}\left[\omega-\left(\alpha-\omega_{c}\right)\right]^{2}\left|H_{\sigma}(\omega)\right|^{2} d \omega\right]^{1 / 2} \\
& \cdot\left[\frac{1}{2 \pi} \int_{R}\left(|\omega|^{2 k-1}+|\alpha|^{2 k-1}\right)^{2}\left|H_{\sigma}\left(\omega-\omega_{c}\right)\right|^{2} d \omega\right]^{1 / 2} \\
= & 4 k \sigma \Phi_{\max } V_{H}^{(1)}\left[\left(\alpha-\omega_{c}\right) / \sigma\right] \cdot\left[\frac { 1 } { 2 \pi } \int _ { R } \left(|\omega|^{2 k-1}\right.\right. \\
& \left.\left.+|\alpha|^{2 k-1}\right)^{2}\left|H_{\sigma}\left(\omega-\omega_{c}\right)\right|^{2} d \omega\right]^{1 / 2}
\end{aligned}
$$

where the equality in (C.3) is obtained from (26). Finally, the simple inequality

$$
\left(|\omega|^{2 k-1}+|\alpha|^{2 k-1}\right)^{2} \leq 2\left(\omega^{4 k-2}+\alpha^{4 k-2}\right)
$$

combined with (28) yields

$$
\begin{aligned}
A \leq & 4 \sqrt{2} k \sigma \Phi_{\max } V_{H}^{(1)}\left[\left(\alpha-\omega_{c}\right) / \sigma\right] \cdot\left[\frac { 1 } { 2 \pi } \int _ { R } \left(\omega^{4 k-2}\right.\right. \\
& \left.\left.+\alpha^{4 k-2}\right)\left|H_{\sigma}\left(\omega-\omega_{c}\right)\right|^{2} d \omega\right]^{1 / 2} \\
= & 4 \sqrt{2} k \alpha^{2 k}\left|\frac{\sigma}{\alpha}\right| \Phi_{\max } V_{H}^{(1)}\left(\frac{\alpha-\omega_{c}}{\sigma}\right) \cdot\left\{\left(\frac{\sigma}{\alpha}\right)^{4 k-2}\right. \\
& \left.\cdot\left[V_{H}^{(2 k-1)}\left(\frac{\omega_{c}}{\sigma}\right)\right]^{2 k}+1\right\}^{1 / 2} \\
\leq & 4 \sqrt{2} k \alpha^{2 k}\left|\frac{\sigma}{\alpha}\right| \Phi_{\max } V_{H}^{(1)}\left(\frac{\alpha-\omega_{c}}{\sigma}\right) \cdot\left\{\left|\frac{\sigma}{\alpha}\right|^{2 k-1}\right. \\
& \left.\cdot\left[V_{H}^{(2 k-1)}\left(\frac{\omega_{c}}{\sigma}\right)\right]^{k}+1\right\}
\end{aligned}
$$

where the simple inequality $\sqrt{b^{2}+1} \leq|b|+1$ is used to obtain (C.4). The $k$ th-order spread $V_{H}^{(k)}$ in (C.4) is defined in (26).

In addition,

$$
\begin{aligned}
B & \leq\left.\left.\alpha^{2 k} \frac{\Phi_{\max }}{2 \pi}|1-| \frac{G_{\sigma}(\alpha)}{G_{\sigma}\left(\omega_{c}\right)}\right|^{2}\left|\int_{R}\right| G_{\sigma}(\omega)\right|^{2} d \omega \\
& =\left.2 \alpha^{2 k} \Phi_{\max }|1-| \frac{G_{\sigma}(\alpha)}{G_{\sigma}\left(\omega_{c}\right)}\right|^{2} \mid
\end{aligned}
$$

from (28) and (29). Combining (C.4) and (C.5) yields the bound (44).

\section{ACKNOWLEDGMENTS}

The authors thank the reviewers for their careful reading of the paper, and for providing several excellent comments that improved the quality of the exposition.

\section{REFERENCES}

[1] H. M. Teager, "Some observations on oral air flow during phonation," IEEE Trans. Acoust., Speech, Signal Processing, vol. ASSP28, pp. 599-601, Oct. 1980

[2] H. M. Teager and S. M. Teager, "Evidence for nonlinear speech production mechanisms in the vocal tract." NATO Advanced Study Institute on Speech Production and Speech Modeling, Bonas, France, July 24, 1989. Boston, MA: Kluwer, 1990, pp. 241-261.

[3] P. Maragos, T. F. Quatieri, and J. F. Kaiser, "Speech nonlinearities, modulations, and energy operators," in Proc. IEEE ICASSP '91, Toronto, Ontario, Canada, May 1991.

[4] A. C. Bovik, N. Gopal, T. Emmoth, and A. Restrepo, "Localized measurement of emergent image frequencies of Gabor wavelets," IEEE Trans. Informat. Theory, vol. 38, pp. 691-712, Mar. 1992.

[5] J. F. Kaiser, "On a simple algorithm to calculate the "energy' of a signal." in Proc. IEEE ICASSP '90. Albuquerque, NM, Apr. 1990.

[6] — "On Teager's energy algorithm and its generalization to continuous signals,', in Proc. IEEE Digital Signal Processing Workshop, New Paltz, NY, Sept. 1990.

[7] P. Maragos, T. F. Quatieri, and J. F. Kaiser, "On separating amplitude from frequency modulations using energy operators," in Proc. IEEE ICASSP '92, San Francisco. CA. Mar. 1992 
[8] _- "On amplitude and freuqency demodulation using energy operators," IEEE Trans. Signal Processing, vol. 41, pp. 1532-1550,

[9] Apr. 1993 . "Energy separation in signal modulations with application to speech analysis," Harvard Robotics Laboratory, Harvard University, Cambridge. MA. Tech. Rep. 91-17, Nov. 1991

[10] P. Maragos, A. C. Bovik, and T. F. Quatieri, "A multidimensional energy operator for image processing." in Proc. SPIE Symp. Visual Commun. Image Processing. Boston, MA, Nov. 1992

[11] A. Papoulis, Probability, Random Variables, and Stochastic Processes, First edition. New York: McGraw-Hill, 1965.

[12] K. S. Miller, Multidimensional Gaussian Distributions. New York, Wiley, 1964

[13] L. Isserlis, "On a formula for the product-moment coefficient of any order of a normal frequency distribution in any number of variables, Biomerrika, vol. 12, pp. 134-139, 1918.

[14] A. C. Bovik and P. Maragos, "Conditions for positivity of an energy operator," IEEE Trans. Signal Processing, to appear Feb. 1994.

[15] A. Papoulis. The Fourier Integral and its Applications. New York McGraw-Hill, 1962.

[16] _- "Limits on bandlimited signals," Proc. IEEE, vol. 55, pp 1677-1686, Oct. 1967

[17] J.-B. Martens, "The Hermite transform-Theory," IEEE Trans. Acoust., Speech, Signal Processing, vol. 38, pp. 1595-1606, Sept. 1990.

[18] S. G. Mallat, "Multifrequency channel decompositions of images and wavelet models," IEEE Trans. Acoust. Speech, Signal Processing, vol. 37, pp. 2091-2110, Dec. 1989.

[19] I. Daubechies, "The wavelet transform, time frequency localization and signal analysis," IEEE Trans. Informat. Theory, vol. 36, pp. 961-1005, Sept. 1990.

[20] - , "Orthonormal bases of compactly supported wavelets," Commun. Pure Appl. Math., vol 41, pp. 909-996, 1988

[21] M. B. Ruskai, G. Beylkin, R. R. Coifman, I. Daubechies, S. G. Mallat, et al., Wavelets and Their Applications. Boston: Jones and Bartlett, 1992.

[22] A. Crosier, D. Esteban, and C. Galand, "Perfect channel splitting by use of interpolation, decimation, and tree decomposition techniques," in Proc. ICISS, Patros, Greece. Aug. 1976.

[23] E. P. Simoncelli and E. H. Adelson, "Subband transforms," in J. W. Woods, Ed., Subband Image Coding. Boston: Kluwer, 1991, pp. 143-192.

[24] I. S. Gradshteyn and I. M. Ryzhik. Tables of Integrals, Series, and Products. Orlando, Florida: Academic, 1980

[25] J. N. Little and L. Schure, Signal Processing Toolbox User's Guide, The MathWorks, Ine, 1992.

[26] L. R. Rabiner, J. H. McClellan, and T. W. Parks, "FIR digital filter design techniques using weighted Chebyshev approximation,' Proc. IEEE, vol. 63, pp. 595-610, 1975.

[27] H. M. Hanson, P. Maragos, and A. Potamianos, "A system for finding speech formants and modulations in energy separation," Harvard Robotics Laboratory, Harvard University, Cambridge, MA, Tech. Rep., 1992.

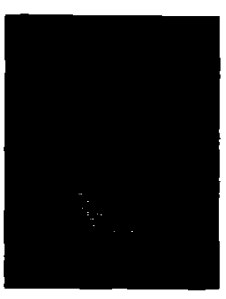

Alan Conrad Bovik ( $\mathrm{S}^{\prime} 80-\mathrm{M}^{\prime} 84-\mathrm{SM}$ '90) was born in Kirkwood, MO on June 25, 1958. He recieved the B.S. degree in Computer Engineering in 1980, and the M.S. and Ph.D. degrees in Electrical and Computer Engineering in 1982 and 1984, respectively, all from the University of I1linois, Urbana-Champaign.

$\mathrm{He}$ is currently the Hartwig Endowed Fellow and Professor in the Department of Electrical and Computer Engineering, the Department of Computer Sciences, and the Biomedical Engineering Program at the University of Texas as Austin, where he is the Director of the Laboratory for Vision Systems. During the Spring of 1992, he held a visiting position in the Division of Applied Sciences, Harvard University, Cambridge, Massachusetts. His current research interests include image processing, computer vision, three-dimensional microscopy, and computational aspects of biological visual perception. He has published over 170 technical articles in these areas, and holds two U.S. patents.

Dr. Bovik is a winner of the University of Texas Engineering Foundation Halliburton Faculty Excellence Award, an Honorable Mention winner of the international Pattern Recognition Society Award for Outstanding Contribution, and was a National Finalist for the 1990 Eta Kappa Nu Outstanding Young Electrical Engineer Award. He has been involved in numerous professional society activities, including: Associate Editor, IEEE Transactions on Signal Processing, 1989-present; Associate Editor of the international journal Pattern Recognition. 1988-present; Editorial Board member for the Journal of Visual Communication and Image Representation, 1993.present; Stecring Committee. IEEE TRAnsactions on IMage Processing, 1992-present: General Chaiman, First IEEE International Conference on Image Processing, to be held in Austin, Texas, in Novem. ber, 1994; Program Chairman, SPIE/SPSE Symposium on Electronic Imaging, February 1990; and Conference Chairman, SPIE Conference on Biomedical lmage Processing, 1990 and 1991 . He is a registered Profes. sional Engineer in the State of Texas and is a frequent consultant to industry and academic institutions.

Petros Maragos (S'81-M'85-SM'91), for a photograph and biography, see page 1550 of the April 1993 issue of this Transactions.

Thomas F, Quatieri (S'73-M'79-SM'87), for a photograph and biography, see page 1550 of the April 1993 issue of this TRANSACTIONS 